JOURNAL OF THE

AMERICAN MATHEMATICAL SOCIETY

Volume 25, Number 1, January 2012, Pages 123-150

S 0894-0347(2011)00714-5

Article electronically published on August 1,2011

\title{
ALGEBRAIC INDEPENDENCE OF PERIODS AND LOGARITHMS OF DRINFELD MODULES
}

\author{
CHIEH-YU CHANG AND MATTHEW A. PAPANIKOLAS, \\ WITH AN APPENDIX BY BRIAN CONRAD
}

\section{INTRODUCTION}

1.1. Drinfeld logarithms. In this paper we prove algebraic independence results about periods, quasi-periods, logarithms, and quasi-logarithms on Drinfeld modules, which are inspired by conjectures from the theory of elliptic curves and abelian varieties. More specifically, let $E$ be an elliptic curve defined over the algebraic numbers $\overline{\mathbb{Q}}$ with Weierstrass $\wp$-function $\wp(z)$. If $E$ does not have complex multiplication, then one expects that the periods $\omega_{1}, \omega_{2}$ and quasi-periods $\eta_{1}, \eta_{2}$ of $E$ are algebraically independent over $\overline{\mathbb{Q}}$; if $E$ has $\mathrm{CM}$, one knows by a theorem of Chudnovsky that their transcendence degree is 2 over $\overline{\mathbb{Q}}$, which also aligns with expectations. Furthermore, if $u_{1}, \ldots, u_{n}$ are complex numbers with $\wp\left(u_{i}\right) \in \overline{\mathbb{Q}} \cup\{\infty\}$ that are linearly independent over $\operatorname{End}(E)$, then one expects that $u_{1}, \ldots, u_{n}$ are algebraically independent over $\overline{\mathbb{Q}}$. Now the $\overline{\mathbb{Q}}$-linear relations among the elliptic logarithms or general abelian logarithms of algebraic points have been studied extensively in the past several decades, but algebraic independence of these logarithms is still wide open (see 3, 24). In the present paper we prove complete analogues of these conjectures for Drinfeld modules of arbitrary rank over general base rings.

Let $\mathbb{F}_{q}$ be a finite field of $q$ elements in characteristic $p$. Let $\mathrm{X}$ be a smooth, projective, geometrically connected curve over $\mathbb{F}_{q}$. We fix a closed point $\infty$ on $X$ and denote by $A$ the ring of functions on $\mathrm{X}$ regular away from $\infty$. Let $k$ be the function field of $\mathrm{X}$ over $\mathbb{F}_{q}$, and let $k_{\infty}$ be the completion of $k$ at $\infty$. Let $\mathbb{C}_{\infty}$ be the completion of a fixed algebraic closure of $k_{\infty}$ at $\infty$, and let $\bar{k}$ be the algebraic closure of $k$ in $\mathbb{C}_{\infty}$.

In Drinfeld's [1] seminal work on elliptic modules, one lets $\Lambda \subseteq \mathbb{C}_{\infty}$ be an $A$ lattice of projective rank $r$, i.e., a finitely generated discrete $A$-module in $\mathbb{C}_{\infty}$, and then forms the exponential function $e_{\Lambda}(z):=z \prod_{0 \neq \lambda \in \Lambda}(1-z / \lambda)$. The Drinfeld $A$-module $\rho^{\Lambda}$ of rank $r$ associated to $\Lambda$ is the $\mathbb{F}_{q}$-linear ring homomorphism from $A$ into the endomorphism ring of the additive group $\mathbb{G}_{\mathrm{a}} / \mathbb{C}_{\infty}$ that satisfies $e_{\Lambda}(a z)=$ $\rho_{a}^{\Lambda}\left(e_{\Lambda}(z)\right)$ for all $a \in A$. Thus $\rho^{\Lambda}$ induces an $A$-module structure on $\mathbb{C}_{\infty}$, which is isomorphic to $\mathbb{C}_{\infty} / \Lambda$ via $e_{\Lambda}(z)$.

Received by the editors May 27, 2010 and, in revised form, May 23, 2011.

2010 Mathematics Subject Classification. Primary 11J93; Secondary 11G09, 11J89.

Key words and phrases. Algebraic independence, Drinfeld modules, periods, logarithms.

The first author was supported by an NCTS postdoctoral fellowship.

The second author was supported by NSF Grant DMS-0903838.

The author of the appendix was supported by NSF grant DMS-0917686.

(C)2011 American Mathematical Society Reverts to public domain 28 years from publication 
We fix a rank $r$ Drinfeld $A$-module $\rho=\rho^{\Lambda}$ that is defined over $\bar{k}$; i.e., as a polynomial in the Frobenius endomorphism, the coefficients of each $\rho_{a}$ are in $\bar{k}$. Let $\exp _{\rho}(z):=e_{\Lambda}(z)$. We set $\operatorname{End}(\rho):=\left\{x \in \mathbb{C}_{\infty} \mid x \Lambda \subseteq \Lambda\right\}$, which can be identified with the endomorphism ring of $\rho$, and let $K_{\rho}$ be its fraction field.

In analogy with the classical results of Schneider and Siegel on the transcendence of elliptic logarithms of algebraic points, Yu 25. proved the transcendence of nonzero elements $u \in \mathbb{C}_{\infty}$ with $\exp _{\rho}(u) \in \bar{k}$. Yu [27] established further in this context the full analogue of Baker's celebrated theorem on the linear independence of logarithms of algebraic numbers. Our first main result is stated as follows.

Theorem 1.1.1. Let $\rho$ be a Drinfeld $A$-module defined over $\bar{k}$. Let $u_{1}, \ldots, u_{n} \in \mathbb{C}_{\infty}$ satisfy $\exp _{\rho}\left(u_{i}\right) \in \bar{k}$ for $i=1, \ldots, n$. If $u_{1}, \ldots, u_{n}$ are linearly independent over $K_{\rho}$, then they are algebraically independent over $\bar{k}$.

Previous work of the authors [7, 17] has established special cases of this theorem when $A$ is a polynomial ring and, either $\rho$ has rank 1, i.e., the Carlitz module case, or $\rho$ has rank 2 without $\mathrm{CM}$ and the characteristic is odd.

1.2. The period matrix and quasi-periodic functions. Based on the analogy between Drinfeld modules and elliptic curves, Anderson, Deligne, Gekeler, and Yu developed a theory of quasi-periodic functions for $\rho$ and defined the de Rham group $H_{\mathrm{DR}}(\rho)$ (see [13, 26]). The group $H_{\mathrm{DR}}(\rho)$ is an $r$-dimensional vector space over $\mathbb{C}_{\infty}$ that parametrizes extensions of $\rho$ by $\mathbb{G}_{\mathrm{a}}$. Associated to each $\delta \in H_{\mathrm{DR}}(\rho)$ there is the entire quasi-periodic function $F_{\delta}(z)$, which sets up the de Rham isomorphism (see [13])

$$
\delta \mapsto\left(\omega \mapsto F_{\delta}(\omega)\right): H_{\mathrm{DR}}(\rho) \stackrel{\sim}{\rightarrow} \operatorname{Hom}_{A}\left(\Lambda, \mathbb{C}_{\infty}\right)
$$

See 3.1 for details about the de Rham group and quasi-periodic functions.

Fixing a maximal $k$-linearly independent set $\left\{\omega_{1}, \ldots, \omega_{r}\right\}$ of $\Lambda$ and a basis $\left\{\delta_{1}, \ldots, \delta_{r}\right\}$ of $H_{\mathrm{DR}}(\rho)$ defined over $\bar{k}$, the $r \times r$ matrix $\mathrm{P}_{\rho}:=\left(F_{\delta_{j}}\left(\omega_{i}\right)\right)$ is a period matrix of $\rho$. Although the matrix $\mathrm{P}_{\rho}$ is not uniquely defined, the field $\bar{k}\left(\mathrm{P}_{\rho}\right)$ is independent of the choices made. Much as in the case of elliptic curves, $\delta_{1}, \ldots, \delta_{r}$ can be chosen appropriately so that the first column of $\mathrm{P}_{\rho}$ consists of elements of $\Lambda$ (periods of the first kind) and the entries of the remaining columns are called quasi-periods (periods of the second kind).

Each entry of $\mathrm{P}_{\rho}$ is transcendental over $k$ by the work of $\mathrm{Yu}$ 25, 26. Let $s$ be the degree of $K_{\rho}$ over $k$. Using Yu's sub-t-module theorem [27, Thm. 0.1], Brownawell proved that all the $\bar{k}$-linear relations among the entries of $\mathrm{P}_{\rho}$ are those induced from endomorphisms of $\rho$; in particular, the dimension of the $\bar{k}$-vector space spanned by the entries of $\mathrm{P}_{\rho}$ is $r^{2} / s$ (cf. [4, Prop. 2]). The period conjecture of Brownawell-Yu asserts that among the entries of $\mathrm{P}_{\rho}$, these linear relations account for all the $\bar{k}$ algebraic relations. The second main theorem of the present paper is to prove this assertion.

Theorem 1.2.2. Let $\rho$ be a Drinfeld A-module of rank $r$ defined over $\bar{k}$, and let $s$ be the degree of $K_{\rho}$ over $k$. Let $\mathrm{P}_{\rho}$ be the period matrix of $\rho$. Then we have

$$
\operatorname{tr} . \operatorname{deg}_{\bar{k}} \bar{k}\left(\mathrm{P}_{\rho}\right)=r^{2} / s
$$


Special cases of this result in rank 2 were previously established by Thiery [23] (algebraic independence in the CM case), David and Denis 9] (quadratic independence), and the authors [7] (algebraic independence in the non-CM, odd characteristic case). Based on this theorem, we further prove the following algebraic independence result on Drinfeld quasi-logarithms. Theorem 1.2.3 has applications to algebraic independence results about periods of the first, second, and third kind for rank 2 Drinfeld modules $[6]$.

Theorem 1.2.3. Let $\rho$ be a Drinfeld A-module of rank $r$ defined over $\bar{k}$. Let $\delta_{1}, \ldots, \delta_{r}$ be a basis of $H_{\mathrm{DR}}(\rho)$ defined over $\bar{k}$. Let $u_{1}, \ldots, u_{n} \in \mathbb{C}_{\infty}$ satisfy $\exp _{\rho}\left(u_{i}\right)$ $\in \bar{k}$ for $i=1, \ldots, n$. If $u_{1}, \ldots, u_{n}$ are linearly independent over $K_{\rho}$, then the $r n$ quasi-logarithms $\bigcup_{i=1}^{n} \bigcup_{j=1}^{r}\left\{F_{\delta_{j}}\left(u_{i}\right)\right\}$ are algebraically independent over $\bar{k}$.

1.3. Outline. To prove these results we travel the route along the deep connection between Drinfeld modules and Anderson's theory of $t$-motives [1]. One of our primary tools is the main theorem of [17, which asserts that the dimension of the Tannakian Galois group of a $t$-motive, defined as the Galois group of a system of difference equations, is equal to the transcendence degree of its associated period matrix (see $₫ 2$ for relevant background). This theorem itself is rooted in a linear independence criterion developed by Anderson, Brownawell, and the second author 2]. Thus the overall strategy for proving each of Theorems 1.1.1, 1.2.2, and 1.2.3 is to create a suitable $t$-motive such that the special values in question are related to its period matrix and then calculate its associated Galois group.

One of our goals in writing this paper has been to establish these algebraic independence results as explicitly as possible, beyond what is supplied in the general theory of [17. If one is purely interested in only the transcendence degree of these values, then it suffices by [17 to calculate the dimension of the Galois group of the $t$-motive. Then using upper bounds on the transcendence degree coming from wellknown $\bar{k}$-linear relations among periods, quasi-periods, and logarithms of Drinfeld modules, one can in principle obtain the results of the present paper simply from the dimension of the Galois group. However, by keeping track of detailed information about these $t$-motives and the solutions of their associated difference equations, we are able to (1) compute the associated Galois groups explicitly, and (2) demonstrate explicitly the connection between these $t$-motives and the known $K_{\rho}$-linear relations among periods and logarithms (see $\S \S 4$ 5). Thus, we recover linear independence results of Brownawell [4] and $\mathrm{Yu}$ [27] in the process, but without the need to appeal to the theory of $t$-modules as Yu's sub-t-module theorem requires. Furthermore, the techniques of the proof are more robust than the ones presented in [7, where the case of rank 2 Drinfeld $\mathbb{F}_{q}[t]$-modules, without $\mathrm{CM}$ and in odd characteristic, was considered. We have endeavored to highlight the advances beyond [7] in this paper.

Throughout this paper we first consider the case when $A$ is a polynomial ring, and then extend the results to general $A$ via complex multiplication. In $\$ 3$, we study the $t$-motive $M_{\rho}$ associated to a given Drinfeld module $\rho$ and its Galois group $\Gamma_{M_{\rho}}$. The main result of $₫ 3$ is to use Anderson generating functions to prove that the image of the Galois representation on the $t$-adic Tate module of $\rho$ is naturally contained inside the $t$-adic valued points of $\Gamma_{M_{\rho}}$. Therefore, using a fundamental theorem of Pink [20] on the openness of the image of the Galois representation on 
the $t$-adic Tate module of $\rho$, we obtain an explicit description of $\Gamma_{M_{\rho}}$ (Theorem 3.5.4). This enables us to prove Theorem 1.2.2

Let $\mathcal{T}$ denote the category of $t$-motives. Given $u_{1}, \ldots, u_{n}$ as in Theorems 1.1.1 and 1.2 .3 , in $\$ 4$ we construct $t$-motives $X_{1}, \ldots, X_{n}$ representing classes in $\operatorname{Ext}_{\mathcal{T}}^{1}\left(\mathbf{1}, M_{\rho}\right)$ such that the union of the entries of their period matrices contains $\bigcup_{i=1}^{n} \bigcup_{j=1}^{r}\left\{F_{\delta_{j}}\left(u_{i}\right)\right\}$. If $u_{1}, \ldots, u_{n}$ together with a $K_{\rho}$-basis of $k \otimes_{A} \Lambda_{\rho}$ are linearly independent over $K_{\rho}$, then we use techniques of Frobenius difference equations to prove that $X_{1}, \ldots, X_{n}$ are $\operatorname{End}_{\mathcal{T}}\left(M_{\rho}\right)$-linearly independent in $\operatorname{Ext}_{\mathcal{T}}^{1}\left(1, M_{\rho}\right)$ (Theorem 4.2.2). As observed by Hardouin 15, the $\operatorname{End}_{\mathcal{T}}\left(M_{\rho}\right)$-linear independence of $X_{1}, \ldots, X_{n}$ provides information for the dimension of the Galois group of $\bigoplus_{i=1}^{n} X_{i}$. Based on Theorem 4.2.2, in 5.1 we give a detailed proof of Theorem 1.2 .3 in the polynomial ring case (Corollary 5.1.6). Using Theorem 3.5.4 and Corollary 5.1.6 we then prove the general cases of Theorems 1.1.1, 1.2.2, and 1.2 .3 in $\$ 5.2$

Note that in an earlier version of this paper, Theorem 1.2 .3 was worked out by the authors under the assumption that $K_{\rho}$ is separable over $k$. This separability hypothesis boiled down to a question on algebraic groups (cf. Lemma 5.1.3), and the purpose of Appendix $\mathrm{A}$ provided by B. Conrad is to remove the hypothesis of separability.

\section{2. $t$-MOTIVES AND DIFFERENCE GALOIS GROUPS}

2.1. Notation and preliminaries. Until 95, where we treat the case of general $A$, we will restrict our base ring $A$ to be the polynomial ring. We adopt the following notation:

$$
\begin{array}{ll}
\mathbb{F}_{q} & =\text { the finite field with } q \text { elements, for } q \text { a power of a prime number } p . \\
\theta, t, z & =\text { independent variables. } \\
A & =\mathbb{F}_{q}[\theta], \text { the polynomial ring in } \theta \text { over } \mathbb{F}_{q} . \\
k & =\mathbb{F}_{q}(\theta), \text { the fraction field of } A . \\
k_{\infty} & =\mathbb{F}_{q}((1 / \theta)), \text { the completion of } k \text { with respect to the place at infinity. } \\
\bar{k}_{\infty} & =\text { a fixed algebraic closure of } k_{\infty} . \\
\bar{k} & =\text { the algebraic closure of } k \text { in } \overline{k_{\infty}} . \\
\mathbb{C}_{\infty} & =\text { the completion of } \overline{k_{\infty}} \text { with respect to the canonical extension of } \infty . \\
A & =\mathbb{F}_{q}[t], \text { the polynomial ring in } t \text { over } \mathbb{F}_{q} . \\
k & =\mathbb{F}_{q}(t), \text { the fraction field of } A . \\
\mathbb{T} & =\left\{f \in \mathbb{C}_{\infty} \llbracket t \rrbracket \mid f \text { converges on }|t|_{\infty} \leq 1\right\}, \text { the Tate algebra over } \mathbb{C}_{\infty} . \\
\mathbb{L} & =\text { the fraction field of } \mathbb{T} \text {. } \\
\mathrm{GL}_{r} / F & =\text { for a field } F, \text { the } F \text {-group scheme of invertible } r \times r \text { matrices. }
\end{array}
$$

For $n \in \mathbb{Z}$, given a Laurent series $f=\sum_{i} a_{i} t^{i} \in \mathbb{C}_{\infty}((t))$, we define the $n$-fold twist of $f$ by $f^{(n)}=\sum_{i} a_{i}^{q^{n}} t^{i}$. For each $n$, the twisting operation is an automorphism of $\mathbb{C}_{\infty}((t))$ and stabilizes several subrings, e.g., $\bar{k} \llbracket t \rrbracket, \bar{k}[t], \mathbb{T}$, and $\mathbb{L}$. For any matrix $B$ with entries in $\mathbb{C}_{\infty}((t))$, we define $B^{(n)}$ by the rule $B^{(n)}{ }_{i j}=B_{i j}^{(n)}$. Also we note (cf. [17, Lem. 3.3.2]) that

$$
\mathbb{F}_{q}[t]=\left\{f \in \mathbb{T} \mid f^{(-1)}=f\right\}, \quad \mathbb{F}_{q}(t)=\left\{f \in \mathbb{L} \mid f^{(-1)}=f\right\} .
$$

Given a ring $R \subseteq \mathbb{C}_{\infty}((t))$ that is invariant under $n$-fold twisting for all $n$, we define twisted polynomial rings $R[\sigma], R\left[\sigma^{-1}\right]$, and $R\left[\sigma, \sigma^{-1}\right]$, subject to the relations

$$
\sigma^{i} f=f^{(-i)} \sigma^{i}, \quad f \in R, i \in \mathbb{Z} .
$$


As a matter of notation, we will often write $\tau$ for $\sigma^{-1}$, and so $R[\tau]=R\left[\sigma^{-1}\right]$. If $R$ itself is a polynomial ring in $t$, say $R=S[t]$, then we will write $R[\sigma]=S[t, \sigma]$ instead of $S[t][\sigma]$.

2.2. Pre- $t$-motives and $t$-motives. We briefly review the definitions and results we will need about pre-t-motives and $t$-motives. The reader is directed to [17, $\S 3]$ for more details. A pre-t-motive $M$ is a left $\bar{k}(t)\left[\sigma, \sigma^{-1}\right]$-module that is finite dimensional over $\bar{k}(t)$. If $\mathbf{m} \in \operatorname{Mat}_{r \times 1}(M)$ is a $\bar{k}(t)$-basis of $M$, then there is a matrix $\Phi \in \mathrm{GL}_{r}(\bar{k}(t))$ so that $\sigma \mathbf{m}=\Phi \mathbf{m}$. We say that $M$ is rigid analytically trivial if there exists $\Psi \in \mathrm{GL}_{r}(\mathbb{L})$ so that

$$
\Psi^{(-1)}=\Phi \Psi
$$

If we let $\sigma$ act diagonally on $\mathbb{L} \otimes_{\bar{k}(t)} M$ and let $M^{\mathrm{B}}$ be the k-subspace fixed by $\sigma$, then $M$ is rigid analytically trivial if and only if $\operatorname{dim}_{\mathrm{k}} M^{\mathrm{B}}=r$. In this case, the entries of $\Psi^{-1} \mathbf{m}$ form a k-basis of $M^{\mathrm{B}}$ [17, Prop. 3.4.7]. By [17, Thm. 3.3.15] the category of rigid analytically trivial pre-t-motives, denoted by $\mathcal{R}$, is a neutral Tannakian category over $\mathrm{k}$ with fiber functor $M \mapsto M^{\mathrm{B}}$ (see [10, $\left.\S \mathrm{II}\right]$ for more details on Tannakian categories). Its trivial object is denoted by $\mathbf{1}$. For a pre-tmotive $M \in \mathcal{R}$, we let $\mathcal{R}_{M}$ denote the strictly full Tannakian subcategory generated by $M$. In this way, $\mathcal{R}_{M}$ is equivalent to the category of representations over $\mathrm{k}$ of an affine algebraic group scheme $\Gamma_{M}$ over k, i.e., $\mathcal{R}_{M} \approx \operatorname{Rep}\left(\Gamma_{M}, \mathrm{k}\right)$. The group $\Gamma_{M}$ is called the Galois group of $M$.

Following [1, 2, 17, an Anderson t-motive $M$ is a left $\bar{k}[t, \sigma]$-module which is free and finitely generated as both a left $\bar{k}[t]$-module and a left $\bar{k}[\sigma]$-module and which satisfies, for $n$ sufficiently large, $(t-\theta)^{n} \mathrm{M} \subseteq \sigma \mathrm{M}$. The functor

$$
\mathrm{M} \mapsto \bar{k}(t) \otimes_{\bar{k}[t]} \mathrm{M}
$$

from Anderson $t$-motives to pre-t-motives is fully faithful up to isogeny (see 17, Thm. 3.4.9]), and if $\bar{k}(t) \otimes_{\bar{k}[t]} \mathrm{M}$ is rigid analytically trivial, then for a $\bar{k}[t]$-basis $\mathbf{m}$ of $M$ it is possible to pick a rigid analytic trivialization $\Psi$ that lies in $\mathrm{GL}_{r}(\mathbb{T})$ (see [17, Prop. 3.4.7]). By definition, the category $\mathcal{T}$ of $t$-motives is the strictly full Tannakian subcategory generated by Anderson $t$-motives in the category of rigid analytically trivial pre-t-motives.

2.3. Galois groups and difference equations. Following [17, $\S \S 4-5]$, the Galois groups of $t$-motives can be constructed explicitly using the theory of Frobenius semi-linear difference equations. Specifically, suppose we are given a triple of fields $F \subseteq K \subseteq L$ together with an automorphism $\sigma_{*}: L \rightarrow L$ that satisfy (1) $\sigma_{*}$ restricts to an automorphism of $K$ and is the identity on $F$; (2) $F=K^{\sigma_{*}}=L^{\sigma_{*}}$; (3) $L$ is a separable extension of $K$. Then given $\Phi \in \mathrm{GL}_{r}(K), \Psi \in \mathrm{GL}_{r}(L)$ satisfying $\sigma_{*} \Psi=\Phi \Psi$, we let $Z_{\Psi}$ be the smallest closed subscheme of $\mathrm{GL}_{r} / K$ that contains $\Psi$ as an $L$-rational point. That is, if $K[X, 1 / \operatorname{det} X], X=\left(X_{i j}\right)$, is the coordinate ring of $\mathrm{GL}_{r} / K$, then the defining ideal of $Z_{\Psi}$ is the kernel of the $K$ algebra homomorphism

$$
X_{i j} \mapsto \Psi_{i j}: K[X, 1 / \operatorname{det} X] \rightarrow L .
$$

Then if we let $\Gamma_{\Psi}$ be the smallest closed subscheme of $\mathrm{GL}_{r} / F$ so that $\Gamma_{\Psi}(\bar{L}) \supseteq$ $\Psi^{-1} Z_{\Psi}(\bar{L})$, the following properties hold. 
Theorem 2.3.1 (Papanikolas [17, Thm. 4.2.11, Thm. 4.3.1]). The scheme $\Gamma_{\Psi}$ is a closed $F$-subgroup scheme of $\mathrm{GL}_{r} / F$, and the closed $K$-subscheme $Z_{\Psi}$ of $\mathrm{GL}_{r} / K$ is stable under right-multiplication by $K \times_{F} \Gamma_{\Psi}$ and is a $\left(K \times_{F} \Gamma_{\Psi}\right)$-torsor, and in particular,

$$
\Gamma_{\Psi}(\bar{L})=\Psi^{-1} Z_{\Psi}(\bar{L}) \text {. }
$$

In addition, if $K$ is algebraically closed in $K(\Psi) \subseteq L$, then

(a) The $K$-scheme $Z_{\Psi}$ is smooth and geometrically connected.

(b) The F-scheme $\Gamma_{\Psi}$ is smooth and geometrically connected.

(c) The dimension of $\Gamma_{\Psi}$ over $F$ is equal to the transcendence degree of $K(\Psi)$ over $K$.

Finally we return to $t$-motives. Suppose that $M \in \mathcal{R}$, that $\Phi \in \mathrm{GL}_{r}(\bar{k}(t))$ represents multiplication by $\sigma$ on $M$, and that $\Psi \in \mathrm{GL}_{r}(\mathbb{L})$ satisfies $\Psi^{(-1)}=\Phi \Psi$. Then using the triple of fields $(F, K, L)=\left(\mathbb{F}_{q}(t), \bar{k}(t), \mathbb{L}\right)$, we can construct the group $\Gamma_{\Psi}$, which is a subgroup of $\mathrm{GL}_{r} / \mathbb{F}_{q}(t)$. Given any object $N$ of $\mathcal{T}_{M}$, one can construct a canonical representation of $\Gamma_{\Psi}$ on $N^{\mathrm{B}}$ (see [17, §4.5]). This permits the identification of $\Gamma_{M}$ and $\Gamma_{\Psi}$.

Theorem 2.3.2 (Papanikolas [17, Thm. 4.5.10]). Given a pre-t-motive $M$ together with $\Phi, \Psi$ as in the paragraph above, the evident functor

$$
N \mapsto N^{\mathrm{B}}: \mathcal{R}_{M} \rightarrow \operatorname{Rep}\left(\Gamma_{\Psi}, \mathbb{F}_{q}(t)\right)
$$

is an equivalence of Tannakian categories. Moreover, $\Gamma_{M} \cong \Gamma_{\Psi}$ over $\mathbb{F}_{q}(t)$.

Furthermore, if $M=\bar{k}(t) \otimes_{\bar{k}[t]} \mathrm{M}$ for an Anderson $t$-motive $\mathrm{M}$, then by [17, Prop. 3.3.9] we can choose $\Psi$ to be in $\mathrm{GL}_{r}(\mathbb{T})$ so that the entries of $\Psi$ converge on all of $\mathbb{C}_{\infty}$ [2, Prop. 3.1.3]. The main theorem of [17] is then the following.

Theorem 2.3.3 (Papanikolas [17, Thm. 1.1.7]). Let $M$ be a t-motive and let $\Gamma_{M}$ be its Galois group. Suppose that $\Phi \in \mathrm{GL}_{r}(\bar{k}(t)) \cap \operatorname{Mat}_{r}(\bar{k}[t])$ represents multiplication by $\sigma$ on $M$ and that $\operatorname{det} \Phi=c(t-\theta)^{s}, c \in \bar{k}^{\times}$. If $\Psi \in \mathrm{GL}_{r}(\mathbb{T})$ is a rigid analytic trivialization for $\Phi$, then tr. $\operatorname{deg}_{\bar{k}} \bar{k}(\Psi(\theta))=\operatorname{dim} \Gamma_{M}$.

\section{Algebraic IndePendence of Periods And QUASI-Periods}

In order to maintain consistency between the notation of Drinfeld modules and $t$-motives, we switch slightly from $\$ 1$ and discuss "Drinfeld A-modules" instead of "Drinfeld $A$-modules."

3.1. Periods and quasi-periods. We review briefly information about Drinfeld modules. For complete treatments the reader is directed to [14, Ch. 3-4] and [22, Ch. 2]. A Drinfeld A-module $\rho$ is defined to be an $\mathbb{F}_{q}$-algebra homomorphism $\rho: \mathrm{A} \rightarrow \mathbb{C}_{\infty}[\tau]$ defined so that $\operatorname{Im}(\rho) \nsubseteq \mathbb{C}_{\infty}$, and if $\rho_{a}=a_{0}+a_{1} \tau+\cdots+a_{s} \tau^{s}$, for $a \in \mathrm{A}$, then $a_{0}=a(\theta)$. Thus,

$$
\rho_{t}=\theta+\kappa_{1} \tau+\cdots+\kappa_{r} \tau^{r},
$$

and we say that $r=\operatorname{deg}_{\tau} \rho_{t}$ is the rank of $\rho$. If $\rho(A) \subseteq K[\tau]$ for a field $K \subseteq \mathbb{C}_{\infty}$, then we say that $\rho$ is defined over $K$.

The Drinfeld module $\rho$ provides $\mathbb{C}_{\infty}$ with the structure of an A-module via

$$
a \cdot x=\rho_{a}(x), \quad \forall a \in A, x \in \mathbb{C}_{\infty},
$$


and we let $\left(\mathbb{C}_{\infty}, \rho\right)$ denote $\mathbb{C}_{\infty}$ together with this A-module structure. A morphism of Drinfeld modules $\rho \rightarrow \rho^{\prime}$ is a twisted polynomial $b \in \mathbb{C}_{\infty}[\tau]$ such that $b \rho_{a}=\rho_{a}^{\prime} b$, for all $a \in A$, and in this way $b$ induces an A-module homomorphism $b:\left(\mathbb{C}_{\infty}, \rho\right) \rightarrow$ $\left(\mathbb{C}_{\infty}, \rho^{\prime}\right)$. We call $b$ an isomorphism of $\rho$ if $b \in \mathbb{C}_{\infty}^{\times}$, and we say that $b$ is defined over $\bar{k}$ if $b \in \bar{k}[\tau]$. The A-algebra of all endomorphisms of a Drinfeld module $\rho$ is denoted by $\operatorname{End}(\rho)$.

There is a unique $\mathbb{F}_{q}$-linear power series with coefficients in $\mathbb{C}_{\infty}$,

$$
\exp _{\rho}(z)=z+\sum_{i=1}^{\infty} \alpha_{i} z^{q^{i}}
$$

called the exponential function of $\rho$, which is entire, is surjective on $\mathbb{C}_{\infty}$, and satisfies $\exp _{\rho}(a(\theta) z)=\rho_{a}\left(\exp _{\rho}(z)\right)$, for $a \in A, z \in \mathbb{C}_{\infty}$. The kernel $\Lambda_{\rho} \subseteq \mathbb{C}_{\infty}$ of $\exp _{\rho}$ is a discrete and finitely generated $A$-submodule of $\mathbb{C}_{\infty}$ of rank $r$; elements in $\Lambda_{\rho}$ are called periods of $\rho$. Thus we have an isomorphism of A-modules $\mathbb{C}_{\infty} / \Lambda_{\rho} \cong\left(\mathbb{C}_{\infty}, \rho\right)$, where on the left-hand side $t$ acts by multiplication by $\theta$. Furthermore, the map

$$
\iota=\left(c_{0}+\cdots+c_{m} \tau^{m} \mapsto c_{0}\right): \operatorname{End}(\rho) \rightarrow\left\{c \in \mathbb{C}_{\infty} \mid c \Lambda_{\rho} \subseteq \Lambda_{\rho}\right\}
$$

is an isomorphism. Throughout this paper, we identify $\operatorname{End}(\rho)$ with the image of $\iota$.

In analogy with the de Rham cohomology for elliptic curves, Anderson, Deligne, Gekeler, and Yu developed a de Rham theory for Drinfeld modules, which characterizes isomorphism classes of extensions of Drinfeld modules by $\mathbb{G}_{\mathrm{a}}$ (see [5, 13, 22, 26]). Continuing with our choice of a Drinfeld A-module $\rho$ of rank $r$, an $\mathbb{F}_{q}$-linear map $\delta: A \rightarrow \mathbb{C}_{\infty}[\tau] \tau$ is called a biderivation if $\delta_{a b}=a(\theta) \delta_{b}+\delta_{a} \rho_{b}$ for all $a, b \in A$. The set of all biderivations $D(\rho)$ is a $\mathbb{C}_{\infty}$-vector space. A biderivation $\delta$ is said to be inner if there exists $m \in \mathbb{C}_{\infty}[\tau]$ so that $\delta_{a}=a(\theta) m-m \rho_{a}$ for all $a \in A$, in which case we denote this biderivation by $\delta^{(m)}$. As in [5, 13, 26, we have

$D_{\mathrm{si}}(\rho)=\left\{\delta^{(m)} \mid m \in \mathbb{C}_{\infty}[\tau] \tau\right\}$ (strictly inner), $H_{\mathrm{DR}}(\rho)=D(\rho) / D_{\mathrm{si}}(\rho)$ (de Rham), and $H_{\mathrm{DR}}(\rho)$ is called the de Rham group of $\rho$. that

Given $\delta \in D(\rho)$, there is a unique power series $F_{\delta}(z)=\sum_{i=1}^{\infty} c_{i} z^{q^{i}} \in \mathbb{C}_{\infty} \llbracket z \rrbracket$ so

$$
F_{\delta}(a(\theta) z)-a(\theta) F_{\delta}(z)=\delta_{a}\left(\exp _{\rho}(z)\right), \quad \forall a \in A .
$$

This is called the quasi-periodic function associated to $\delta$. It is an entire function on $\mathbb{C}_{\infty}$ and satisfies $F_{\delta}(z+\omega)=F_{\delta}(z)+F_{\delta}(\omega)$, for all $\omega \in \Lambda_{\rho}$. The values $F_{\delta}(\omega)$ for $\omega \in \Lambda_{\rho}$ are called quasi-periods of $\rho$. Since the map $\left.F_{\delta}\right|_{\Lambda_{\rho}}$ is $A$-linear, there is a well-defined $\mathbb{C}_{\infty}$-linear map,

$$
\delta \mapsto\left(\omega \mapsto F_{\delta}(\omega)\right): H_{\mathrm{DR}}(\rho) \rightarrow \operatorname{Hom}_{A}\left(\Lambda_{\rho}, \mathbb{C}_{\infty}\right),
$$

and this map is an isomorphism (see [13]).

Note that as $A=\mathbb{F}_{q}[t]$, every biderivation is uniquely determined by the image of $t$. Hence the $\mathbb{C}_{\infty}$-vector space $H_{\mathrm{DR}}(\rho)$ has a conveniently chosen basis, represented by biderivations $\delta_{1}, \delta_{2}, \ldots, \delta_{r}: \delta_{1}$ is the inner biderivation $\delta^{(1)}: a \mapsto a(\theta)-\rho_{a}$ that generates the space of all inner biderivations modulo $D_{\mathrm{si}}(\rho)$, and the biderivations $\delta_{2}, \ldots, \delta_{r}$ are defined by $\delta_{i}: t \mapsto \tau^{i-1}$. Now we have $F_{\delta^{(1)}}(z)=z-\exp _{\rho}(z)$, and so $F_{\delta^{(1)}}(\omega)=\omega$ for all $\omega \in \Lambda_{\rho}$. Thus if we put $F_{\tau^{i}}(z):=F_{\delta_{i+1}}(z), i=1, \ldots, r-1$, 
and let $\omega_{1}, \ldots, \omega_{r}$ be an $A$-basis of $\Lambda_{\rho}$, then we can set

$$
\mathrm{P}_{\rho}:=\left(F_{\delta_{j}}\left(\omega_{i}\right)\right)=\left(\begin{array}{cccc}
\omega_{1} & F_{\tau}\left(\omega_{1}\right) & \cdots & F_{\tau^{r-1}}\left(\omega_{1}\right) \\
\omega_{2} & F_{\tau}\left(\omega_{2}\right) & \cdots & F_{\tau^{r-1}}\left(\omega_{2}\right) \\
\vdots & \vdots & & \vdots \\
\omega_{r} & F_{\tau}\left(\omega_{r}\right) & \cdots & F_{\tau^{r-1}}\left(\omega_{r}\right)
\end{array}\right),
$$

which we refer to as the period matrix of $\rho$. The first column contains periods (of the first kind) of $\rho$, while the remaining columns contain quasi-periods (periods of the second kind). Basic properties of biderivations show that the field $\bar{k}\left(\mathrm{P}_{\rho}\right)$ depends only on the isomorphism class of $\rho$ and not on the choice of basis for $\Lambda_{\rho}$ or even the choice of basis for $H_{\mathrm{DR}}(\rho)$ defined over $\bar{k}$ (i.e., $\left.\delta(\mathrm{A}) \subseteq \bar{k}[\tau] \tau\right)$.

3.2. The $t$-adic Tate module and Anderson generating functions. For any $a \in \mathrm{A}$ the torsion A-module $\rho[a]:=\left\{x \in \mathbb{C}_{\infty} \mid \rho_{a}(x)=0\right\}$ is isomorphic to $(\mathrm{A} /(a))^{\oplus r}$. Thus if we let $v$ be any monic irreducible polynomial in $\mathrm{A}$, we can define the Tate module $T_{v}(\rho)$ to be

$$
T_{v}(\rho):=\lim _{\longleftarrow} \rho\left[v^{m}\right] \cong A_{v}^{\oplus r} .
$$

Now assume that $\rho$ is defined over $K \subseteq \bar{k}$. Every element of $\rho\left[v^{m}\right]$ is separable over $K$, and so the absolute Galois group $\operatorname{Gal}\left(K^{\text {sep }} / K\right)$ of the separable closure of $K$ inside $\bar{k}$ acts on $T_{v}(\rho)$, thus defining a representation

$$
\varphi_{v}: \operatorname{Gal}\left(K^{\mathrm{sep}} / K\right) \rightarrow \operatorname{Aut}\left(T_{v}(\rho)\right) \cong \mathrm{GL}_{r}\left(\mathrm{~A}_{v}\right) .
$$

Because it is well-suited to our purposes we now specialize to the case that $v=t$. Fixing an $A$-basis $\omega_{1}, \ldots, \omega_{r}$ of $\Lambda_{\rho}$, we define

$$
\xi_{i, m}:=\exp _{\rho}\left(\frac{\omega_{i}}{\theta^{m+1}}\right) \in \rho\left[t^{m+1}\right]
$$

for $1 \leq i \leq r$ and $m \geq 0$. In this way we define an $A_{t}$-basis $x_{1}, \ldots, x_{r}$ of $T_{t}(\rho)$ by taking $x_{i}:=\left(\xi_{i, 0}, \xi_{i, 1}, \xi_{i, 2}, \ldots\right)$. Thus for $\epsilon \in \operatorname{Gal}\left(K^{\text {sep }} / K\right)$ we can define $g_{\epsilon} \in$ $\mathrm{GL}_{r}\left(\mathbb{F}_{q} \llbracket t \rrbracket\right)$ so that

$$
\varphi_{t}(\epsilon) \mathbf{x}=g_{\epsilon} \mathbf{x}
$$

where $\mathbf{x}=\left[x_{1}, \ldots, x_{r}\right]^{\text {tr }}$.

For each $i, 1 \leq i \leq r$, we define an Anderson generating function,

$$
f_{i}(t):=\sum_{m=0}^{\infty} \xi_{i, m} t^{m}=\sum_{m=0}^{\infty} \exp _{\rho}\left(\frac{\omega_{i}}{\theta^{m+1}}\right) t^{m} \in K^{\mathrm{sep}} \llbracket t \rrbracket .
$$

The group $\operatorname{Gal}\left(K^{\mathrm{sep}} / K\right)$ acts on $K^{\mathrm{sep}} \llbracket t \rrbracket$ by acting on each coefficient, and we extend this action entry-wise to matrices with entries in $K^{\mathrm{sep}} \llbracket t \rrbracket$. The following lemma and corollary show that the Galois action on $f_{i}$ and on its Frobenius twists as elements of $K^{\mathrm{sep}} \llbracket t \rrbracket$ is compatible with its action on them as elements of $T_{t}(\rho)$.

Lemma 3.2.2. Let $\mathbf{f}=\left[f_{1}, \ldots, f_{r}\right]^{\text {tr }}$. For any $\epsilon \in \operatorname{Gal}\left(K^{\mathrm{sep}} / K\right)$, we have $\epsilon(\mathbf{f})=$ $g_{\epsilon} \mathbf{f}$, where $\epsilon(\mathbf{f})=\left[\epsilon\left(f_{1}\right), \ldots, \epsilon\left(f_{r}\right)\right]^{\mathrm{tr}}$.

Proof. Given $\mathbf{a}=\sum_{\ell=0}^{\infty} a_{\ell} t^{\ell} \in \mathbb{F}_{q} \llbracket t \rrbracket=\mathrm{A}_{t}$, it is easy to see that for each $i, \mathbf{a} \cdot x_{i}=$ $\left(\mathbf{a} \cdot \xi_{i, 0}, \mathbf{a} \cdot \xi_{i, 1}, \mathbf{a} \cdot \xi_{i, 2}, \ldots\right)$, where for each $m \geq 0$,

$$
\mathbf{a} \cdot \xi_{i, m}=a_{m} \xi_{i, 0}+a_{m-1} \xi_{i, 1}+\cdots+a_{0} \xi_{i, m} \in \rho\left[t^{m+1}\right] .
$$


Now fix any $1 \leq s \leq r$, and let $\left[h_{s, 1}, \ldots, h_{s, r}\right] \in \operatorname{Mat}_{1 \times r}\left(\mathbb{F}_{q} \llbracket t \rrbracket\right)$ be the $s$-th row of $g_{\epsilon}$. Then

$$
\epsilon\left(x_{s}\right)=\varphi_{t}(\epsilon)\left(x_{s}\right)=\sum_{i=1}^{r} h_{s, i} \cdot x_{i} .
$$

Thus if we write $h_{s, i}=\sum_{\ell=0}^{\infty} \gamma_{i, \ell} t^{\ell}$ as an element of $\mathbb{F}_{q} \llbracket t \rrbracket$, we see from (3.2.3) that the $(m+1)$-th entry of $\epsilon\left(x_{s}\right)$ is

$$
\epsilon\left(\xi_{s, m}\right)=\sum_{i=1}^{r}\left(\gamma_{i, m} \xi_{i, 0}+\gamma_{i, m-1} \xi_{i, 1}+\cdots+\gamma_{i, 0} \xi_{i, m}\right) .
$$

It follows that

$$
\epsilon\left(f_{s}\right)=\sum_{m=0}^{\infty}\left(\sum_{i=1}^{r}\left(\gamma_{i, m} \xi_{i, 0}+\gamma_{i, m-1} \xi_{i, 1}+\cdots+\gamma_{i, 0} \xi_{i, m}\right)\right) t^{m} .
$$

By reversing the order of summation we see that $\epsilon\left(f_{s}\right)$ is the same as $\left[h_{s, 1}, \ldots, h_{s, r}\right] \mathbf{f}$ via multiplication of power series in $K^{\mathrm{sep}} \llbracket t \rrbracket$.

Corollary 3.2.4. For $1 \leq i, j \leq r$, define $\Upsilon \in \operatorname{Mat}_{r}\left(K^{\operatorname{sep}} \llbracket t \rrbracket\right)$ so that $\Upsilon_{i j}:=f_{i}^{(j-1)}$. Then for any $\epsilon \in \operatorname{Gal}\left(K^{\mathrm{sep}} / K\right)$,

$$
\epsilon\left(\Upsilon^{(1)}\right)=g_{\epsilon} \Upsilon^{(1)}
$$

Proof. The $j$-th column of $\Upsilon^{(1)}$ is simply $\mathbf{f}^{(j)}$, where $\mathbf{f}$ is defined in Lemma 3.2.2. Since for each $i, j$, we have $\epsilon\left(f_{i}^{(j)}\right)=\epsilon\left(f_{i}\right)^{(j)}$, it follows from Lemma 3.2 .2 that $\epsilon\left(\mathbf{f}^{(j)}\right)=\epsilon(\mathbf{f})^{(j)}=\left(g_{\epsilon} \mathbf{f}\right)^{(j)}$. Since $g_{\epsilon} \in \mathrm{GL}_{r}\left(\mathbb{F}_{q} \llbracket t \rrbracket\right)$, we have $\left(g_{\epsilon} \mathbf{f}\right)^{(j)}=g_{\epsilon} \mathbf{f}^{(j)}$.

3.3. Anderson $t$-motives associated to Drinfeld modules. Let us continue with our choice of a rank $r$ Drinfeld A-module $\rho$, defined as in (3.1.1) over $\bar{k}$. As mentioned in $3.1, \bar{k}\left(P_{\rho}\right)$ is unique up to isomorphisms of $\rho$ and so we assume that $\kappa_{r}=1$. The proofs in this section are essentially identical to those in [7, §2.4], and we omit them for brevity.

We associate an Anderson $t$-motive $M_{\rho}$ to $\rho$ in the following way. We let $M_{\rho}$ be isomorphic to the direct sum of $r$ copies of $\bar{k}[t]$, and we represent multiplication by $\sigma$ on $M_{\rho}$ with respect to the standard basis $m_{1}, \ldots, m_{r}$ of $M_{\rho}$ by

$$
\Phi_{\rho}:=\left(\begin{array}{cccc}
0 & 1 & \cdots & 0 \\
\vdots & \vdots & \ddots & \vdots \\
0 & 0 & \cdots & 1 \\
(t-\theta) & -\kappa_{1}^{(-1)} & \cdots & -\kappa_{r-1}^{(-r+1)}
\end{array}\right) .
$$

Using a similar proof to [7, Lem. 2.4.1] we find that $M_{\rho}$ defines an Anderson $t$ motive. As a $\bar{k}[\sigma]$-module, $\mathrm{M}_{\rho}$ has rank 1 , and in fact $\mathrm{M}_{\rho}=\bar{k}[\sigma] m_{1}$. Finally we let $M_{\rho}:=\bar{k}(t) \otimes_{\bar{k}[t]} M_{\rho}$ be the pre-t-motive associated to $M_{\rho}$.

Now a morphism $b: \rho \rightarrow \rho^{\prime}$ of Drinfeld modules induces a morphism $\beta: M_{\rho} \rightarrow$ $\mathrm{M}_{\rho^{\prime}}$ of Anderson $t$-motives: if $b=\sum c_{i} \tau^{i}$, then letting $b^{*}=\sum c_{i}^{(-i)} \sigma^{i} \in \bar{k}[\sigma]$, it follows, using methods similar to [7, Lem. 2.4.2], that $\beta$ is the $\bar{k}[\sigma]$-linear map such that $\beta\left(m_{1}\right)=b^{*} m_{1}^{\prime}$. Moreover, we have the following crucial result due to Anderson (see [7, Prop. 2.4.3]). 
Proposition 3.3.2. The functor $\rho \mapsto M_{\rho}$ from Drinfeld A-modules over $\bar{k}$ to the category of Anderson t-motives is fully faithful. Moreover, for any Drinfeld module $\rho$ over $\bar{k}$,

$$
\operatorname{End}(\rho) \cong \operatorname{End}_{\bar{k}[t, \sigma]}\left(M_{\rho}\right), \quad K_{\rho} \cong \operatorname{End}_{\mathcal{T}}\left(M_{\rho}\right) .
$$

Corollary 3.3.3. For a Drinfeld A-module $\rho$ over $\bar{k}, M_{\rho}$ is a simple left $\bar{k}(t)\left[\sigma, \sigma^{-1}\right]$ module.

Proof. Every nonzero morphism of Drinfeld A-modules is surjective, and therefore, every object in the category of Drinfeld A-modules is simple. By Proposition 3.3.2. $\mathrm{M}_{\rho}$ is a simple Anderson $t$-motive, and the result follows easily from 2 , Prop. 4.4.10].

3.4. Drinfeld modules and rigid analytic trivializations. Given a Drinfeld A-module $\rho$ as in the previous section, we demonstrate here how its associated Anderson $t$-motive $M_{\rho}$ is rigid analytically trivial. The arguments follow methods of Pellarin [18, §4.2].

For $u \in \mathbb{C}_{\infty}$, we consider the Anderson generating function

$$
f_{u}(t):=\sum_{m=0}^{\infty} \exp _{\rho}\left(\frac{u}{\theta^{m+1}}\right) t^{m}=\sum_{i=0}^{\infty} \frac{\alpha_{i} u^{q^{i}}}{\theta^{q^{i}}-t} \in \mathbb{T},
$$

where $\exp _{\rho}(z)=z+\sum_{i=1}^{\infty} \alpha_{i} z^{q^{i}}$. The function $f_{u}(t)$ is meromorphic on $\mathbb{C}_{\infty}$ with simple poles at $t=\theta, \theta^{q}, \ldots$ with residues $-u,-\alpha_{1} u^{q}, \ldots$. Since $\rho_{t}\left(\exp _{\rho}\left(u / \theta^{m+1}\right)\right)$ $=\exp _{\rho}\left(u / \theta^{m}\right)$,

$$
\kappa_{1} f_{u}^{(1)}(t)+\cdots+\kappa_{r-1} f_{u}^{(r-1)}(t)+f_{u}^{(r)}=(t-\theta) f_{u}(t)+\exp _{\rho}(u) .
$$

It follows upon specializing at $t=\theta$ that

$$
\kappa_{1} f_{u}^{(1)}(\theta)+\cdots+\kappa_{r-1} f_{u}^{(r-1)}(\theta)+f_{u}^{(r)}(\theta)=-u+\exp _{\rho}(u) .
$$

Lemma 3.4.4. If $u_{1}, \ldots, u_{n} \in \mathbb{C}_{\infty}$ are linearly independent over $k$, then the functions $f_{u_{1}}(t), \ldots, f_{u_{n}}(t)$ are linearly independent over $\mathrm{k}$.

Proof. For $c_{1}(t), \ldots, c_{n}(t) \in \mathrm{k}, \operatorname{Res}_{t=\theta} \sum_{i=1}^{n} c_{i}(t) f_{u_{i}}(t)=-\sum_{i=1}^{n} c_{i}(\theta) u_{i}$.

Once we fix an $A$-basis $\omega_{1}, \ldots, \omega_{r}$ of the period lattice $\Lambda_{\rho}$ of $\rho$, the Anderson generating functions $f_{1}, \ldots, f_{r}$ from (3.2.1) are then $f_{\omega_{1}}, \ldots, f_{\omega_{r}}$. For $1 \leq i \leq r$ and $1 \leq j \leq r-1$, it follows from [13, p. 194], [22, §6.4] that

$$
F_{\tau^{j}}\left(\omega_{i}\right)=\sum_{m=0}^{\infty} \exp _{\rho}\left(\frac{\omega_{i}}{\theta^{m+1}}\right)^{q^{j}} \theta^{m}=f_{i}^{(j)}(\theta) .
$$

Define the matrix

$$
\Upsilon=\left(\begin{array}{cccc}
f_{1} & f_{1}^{(1)} & \cdots & f_{1}^{(r-1)} \\
f_{2} & f_{2}^{(1)} & \cdots & f_{2}^{(r-1)} \\
\vdots & \vdots & & \vdots \\
f_{r} & f_{r}^{(1)} & \cdots & f_{r}^{(r-1)}
\end{array}\right)
$$

By Lemma 3.4.4, $f_{1}, \ldots, f_{r}$ are linearly independent over $k$, and so if one argues as in [14, Lem. 1.3.3] (the case of Moore determinants), it follows that $\operatorname{det} \Upsilon \neq 0$. 
Now letting

$$
\Theta=\left(\begin{array}{cccc}
0 & \cdots & 0 & t-\theta \\
1 & \cdots & 0 & -\kappa_{1} \\
\vdots & \ddots & \vdots & \vdots \\
0 & \cdots & 1 & -\kappa_{r-1}
\end{array}\right),
$$

we see from (3.4.2) that $\Upsilon^{(1)}=\Upsilon \Theta$. To create a rigid analytic trivialization for $\Phi_{\rho}$, we let

$$
V:=\left(\begin{array}{ccccc}
\kappa_{1} & \kappa_{2}^{(-1)} & \cdots & \kappa_{r-1}^{(-r+2)} & 1 \\
\kappa_{2} & \kappa_{3}^{(-1)} & \cdots & 1 & \\
\vdots & \vdots & & & \\
\kappa_{r-1} & 1 & & & \\
1 & & & &
\end{array}\right)
$$

and then set

$$
\Psi_{\rho}:=V^{-1}\left[\Upsilon^{(1)}\right]^{-1} .
$$

Since $V^{(-1)} \Phi_{\rho}=\Theta V$ and $\Upsilon^{(1)}=\Upsilon \Theta$, it follows that $\Psi_{\rho}^{(-1)}=\Phi_{\rho} \Psi_{\rho}$. Thus the pre$t$-motive $M_{\rho}$ is rigid analytically trivial and is in the category of $t$-motives (cf. [17, Prop. 3.4.7(c)]).

Proposition 3.4.7. Given a Drinfeld A-module $\rho$ defined over $\bar{k}$, the matrix $\Psi_{\rho}$ defined in (3.4.6) and the period matrix $\mathrm{P}_{\rho}$ defined in (3.1.4) satisfy the following properties:

(a) The entries of $\Psi_{\rho}$ are regular at $t=\theta$.

(b) tr. $\operatorname{deg}_{\bar{k}} \bar{k}\left(\Psi_{\rho}(\theta)\right)=\operatorname{dim} \Gamma_{\Psi_{\rho}}$.

(c) $\bar{k}\left(\Psi_{\rho}(\theta)\right)=\bar{k}\left(\mathrm{P}_{\rho}\right)$.

Proof. By [17, Prop. 3.3.9(c), §4.1.6], there exists a matrix $U \in \mathrm{GL}_{r}(\mathrm{k})$ such that $\widetilde{\Psi}:=\Psi_{\rho} U$ is a rigid analytic trivialization of $\Phi$ and $\widetilde{\Psi} \in \mathrm{GL}_{r}(\mathbb{T})$. By [2, Prop. 3.1.3], the entries of $\widetilde{\Psi}$ converge on all of $\mathbb{C}_{\infty}$. Part (a) then follows since the entries of $\widetilde{\Psi}$ and $U^{-1}$ are all regular at $t=\theta$. Part (b) follows directly from Theorem 2.3.3. since $\bar{k}\left(\Psi_{\rho}(\theta)\right)=\bar{k}(\widetilde{\Psi}(\theta))$. To prove (c) we first observe that $\bar{k}\left(\Psi_{\rho}(\theta)\right)=\bar{k}\left(\Upsilon^{(1)}(\theta)\right)$ from (3.4.6). For $1 \leq i \leq r$ and $1 \leq j \leq r-1$, it follows from (3.4.5) that $\Upsilon^{(1)}(\theta)_{i j}=F_{\tau^{j}}\left(\omega_{i}\right)$. From (3.4.3) and (3.4.5) we see that

$$
\Upsilon^{(1)}(\theta)_{i r}=-\omega_{i}-\sum_{s=1}^{r-1} \kappa_{s} F_{\tau^{s}}\left(\omega_{i}\right),
$$

and thus $\bar{k}\left(\Upsilon^{(1)}(\theta)\right)=\bar{k}\left(\mathrm{P}_{\rho}\right)$.

3.5. The Galois group $\Gamma_{\Psi_{\rho}}$. The following theorem demonstrates an explicit connection between the Galois group $\Gamma_{\Psi_{\rho}}$ of a Drinfeld module $\rho$ arising from difference equations and the $t$-adic representation attached to the Galois action on the torsion points of $\rho$.

Theorem 3.5.1. Let $\rho$ be a Drinfeld A-module that is defined over a field $K \subseteq \bar{k}$ such that $K$ is a finite extension of $k ; \operatorname{End}(\rho) \subseteq K[\tau]$; and $Z_{\Psi_{\rho}}$ is defined over $K(t)$. Then

$$
\varphi_{t}\left(\operatorname{Gal}\left(K^{\mathrm{sep}} / K\right)\right) \subseteq \Gamma_{\Psi_{\rho}}\left(\mathbb{F}_{q}((t))\right)
$$


Proof. Let $\epsilon \in \operatorname{Gal}\left(K^{\mathrm{sep}} / K\right)$, and let $g_{\epsilon} \in \mathrm{GL}_{r}\left(\mathbb{F}_{q} \llbracket t \rrbracket\right)$ be defined as in 33.2 . Then by (3.4.6) and Corollary 3.2.4.

$$
\epsilon\left(\Psi_{\rho}\right)=\epsilon\left(V^{-1}\left[\Upsilon^{(1)}\right]^{-1}\right)=V^{-1}\left[g_{\epsilon} \Upsilon^{(1)}\right]^{-1}=\Psi_{\rho} g_{\epsilon}^{-1} .
$$

Now let $S \subseteq K(t)[X, 1 / \operatorname{det} X]$ denote a finite set of generators of the defining ideal of $Z_{\Psi_{\rho}}$. Thus for any $h \in S$, we have $h\left(\Psi_{\rho}\right)=0$. Since $\epsilon$ fixes the coefficients of $h$, we have

$$
0=\epsilon\left(h\left(\Psi_{\rho}\right)\right)=h\left(\Psi_{\rho} g_{\epsilon}^{-1}\right) .
$$

Therefore, $\Psi_{\rho} g_{\epsilon}^{-1} \in Z_{\Psi_{\rho}}\left(\mathbb{C}_{\infty}((t))\right)$. By Theorem 2.3.1] we see that $g_{\epsilon}^{-1} \in$ $\Gamma_{\Psi_{\rho}}\left(\mathbb{F}_{q}((t))\right)$.

Remark 3.5.3. Using basic properties of Drinfeld modules [14, $\S 4.7]$ and the fact that $Z_{\Psi_{\rho}}$ is of finite type over $\bar{k}(t)$, we can select a field $K \subseteq \bar{k}$ satisfying the properties in the previous theorem for any Drinfeld A-module $\rho$ defined over $\bar{k}$.

Combining Theorem 3.5.1 with theorems of Pink 20] on the image of Galois representations attached to Drinfeld modules, we calculate $\Gamma_{\Psi_{\rho}}$ exactly. It is also possible to identify the Galois group $\Gamma_{\Psi_{\rho}}$ with the Hodge-Pink group 19] (e.g., see [16] for additional applications to pure $t$-motives), and we thank U. Hartl for pointing out the proper references to us. However, here we have a direct proof suited to our context.

Theorem 3.5.4. Let $\rho$ be a Drinfeld A-module of rank $r$ defined over $\bar{k}$. Set $\mathrm{K}_{\rho}:=\operatorname{End}_{\mathcal{T}}\left(M_{\rho}\right)$, which embeds naturally into $\operatorname{End}\left(M_{\rho}^{\mathrm{B}}\right)=\operatorname{Mat}_{r}(\mathrm{k})$ by Theorem 2.3.2. Define the centralizer $\operatorname{Cent}_{\mathrm{GL}_{r} / \mathrm{k}}\left(\mathrm{K}_{\rho}\right)$ to be the algebraic group over $\mathrm{k}$ such that for any $\mathrm{k}$-algebra $\mathrm{R}$,

$$
\operatorname{Cent}_{\mathrm{GL}_{r} / \mathrm{k}}\left(\mathrm{K}_{\rho}\right)(\mathrm{R}):=\left\{\gamma \in \mathrm{GL}_{r}(\mathrm{R}) \mid \gamma g=g \gamma \text { for all } g \in \mathrm{R} \otimes_{\mathrm{K}} \mathrm{K}_{\rho} \subseteq \operatorname{Mat}_{r}(\mathrm{R})\right\} .
$$

Then

$$
\Gamma_{\Psi_{\rho}}=\operatorname{Cent}_{\mathrm{GL}_{r} / \mathrm{k}}\left(\mathrm{K}_{\rho}\right) .
$$

In particular, tr. $\operatorname{deg}_{\bar{k}} \bar{k}\left(\mathrm{P}_{\rho}\right)=r^{2} / s$, where $s=\left[\mathrm{K}_{\rho}: \mathrm{k}\right]$.

Proof. By general Tannakian theory [10, §II], the tautological representation $\Gamma_{M} \hookrightarrow$ $\mathrm{GL}\left(M^{\mathrm{B}}\right)$ is functorial in $M$, and so there is a natural embedding

$$
\Gamma_{\Psi_{\rho}} \hookrightarrow \operatorname{Cent}_{\mathrm{GL}_{r} / \mathrm{k}}\left(\mathrm{K}_{\rho}\right) .
$$

By [20, Thm. 0.2], the Zariski closure of $\varphi_{t}\left(\mathrm{Gal}\left(K^{\mathrm{sep}} / K\right)\right)$ inside $\mathrm{GL}_{r}\left(\mathbb{F}_{q}((t))\right)$ is open in $\operatorname{Cent}_{\mathrm{GL}_{r}\left(\mathbb{F}_{q}((t))\right)}\left(\mathrm{K}_{\rho}\right)$ with respect to the $t$-adic topology. Thus from Theorem 3.5 .1 and (3.5.5),

$$
\operatorname{dim} \Gamma_{\Psi_{\rho}}=\operatorname{dim} \operatorname{Cent}_{\mathrm{GL}_{r} / \mathrm{k}}\left(\mathrm{K}_{\rho}\right) .
$$

Since the defining polynomials of $\operatorname{Cent}_{\mathrm{GL}_{r} / \mathrm{k}}\left(\mathrm{K}_{\rho}\right)$ are degree one polynomials, it is connected, and hence $\Gamma_{\Psi_{\rho}}=\operatorname{Cent}_{\mathrm{GL}_{r} / \mathrm{k}}\left(\mathrm{K}_{\rho}\right)$. By Proposition 3.4.7, it now suffices to show that $\operatorname{dim} \Gamma_{\Psi_{\rho}}=r^{2} / s$. Since $\Gamma_{\Psi_{\rho}}$ is smooth by Theorem 2.3.1(b), we have $\operatorname{dim} \Gamma_{\Psi_{\rho}}=\operatorname{dim} \operatorname{Lie} \Gamma_{\Psi_{\rho}}$. Now Lie $\Gamma_{\Psi_{\rho}}=\operatorname{Cent}_{\operatorname{Mat}_{r}(\mathrm{k})}\left(\mathrm{K}_{\rho}\right)$, and the result follows from [12, Thm. 3.15(3)].

Corollary 3.5.6. For any $\mathrm{k}$-algebra $\mathrm{R}$, we have $\Gamma_{\Psi_{\rho}}(\mathrm{R}) \cong \mathrm{GL}_{r / s}\left(\mathrm{R} \otimes_{\mathrm{k}} \mathrm{K}_{\rho}\right)$ naturally in $\mathrm{R}$. 
Proof. Since we have the natural embedding $\mathrm{K}_{\rho} \hookrightarrow \operatorname{End}\left(M_{\rho}^{\mathrm{B}}\right), M_{\rho}^{\mathrm{B}}$ is a $\mathrm{K}_{\rho}$-vector space of dimension $r / s$. As the group of R-valued points of $\operatorname{Cent}_{\mathrm{GL}_{r} / \mathrm{k}}\left(\mathrm{K}_{\rho}\right)$ is identified with $\left\{\phi \in \operatorname{Aut}_{\mathrm{R}}\left(\mathrm{R} \otimes_{\mathrm{K}} M_{\rho}^{\mathrm{B}}\right) \mid \phi\right.$ is $\mathrm{R} \otimes_{\mathrm{K}} \mathrm{K}_{\rho}$-linear $\}$, which itself is identified with $\mathrm{GL}_{r / s}\left(\mathrm{R} \otimes_{\mathrm{K}} \mathrm{K}_{\rho}\right)$, the result follows from Theorem 3.5.4.

Corollary 3.5.7. Every object $W \in \boldsymbol{R} \mathbf{e p}\left(\Gamma_{M_{\rho}}, \mathrm{k}\right)$ is completely reducible.

Proof. Let $k\left[\Gamma_{\Psi_{\rho}}(\mathrm{k})\right] \subseteq \operatorname{Mat}_{r}(\mathrm{k})$ be the k-algebra generated by all elements of $\Gamma_{\Psi}(\mathrm{k})$. Since by Theorem 3.5.4, $\Gamma_{\Psi_{\rho}}(\mathrm{k})=\operatorname{Cent}_{\mathrm{GL}_{r}(\mathrm{k})}\left(\mathrm{K}_{\rho}\right)$, we have $\mathrm{k}\left[\Gamma_{\Psi_{\rho}}(\mathrm{k})\right]=$ $\operatorname{Cent}_{\text {Mat }_{r}(\mathrm{k})}\left(\mathrm{K}_{\rho}\right)$, which is a simple ring (cf. [12, Thm. 3.15]). Regarding $W$ as a module over $\operatorname{Cent}_{\operatorname{Mat}_{r}(\mathrm{k})}\left(\mathrm{K}_{\rho}\right)$, we have a decomposition $W \cong \bigoplus W_{i}$ such that each $W_{i}$ is a simple $\operatorname{Cent}_{\mathrm{Mat}_{r}(\mathrm{k})}\left(\mathrm{K}_{\rho}\right)$-module.

Now it suffices to show that each $W_{i}$ is an object in $\operatorname{Rep}\left(\Gamma_{M_{\rho}}, \mathrm{k}\right)$. To prove it, for any $k$-algebra $\mathrm{R}$ we first observe that $\mathrm{R} \otimes_{\mathrm{k}} W_{i}$ is a module over $\mathrm{R} \otimes_{\mathrm{k}} \operatorname{Cent}_{\mathrm{Mat}_{r}(\mathrm{k})}\left(\mathrm{K}_{\rho}\right)$, which is naturally isomorphic to $\operatorname{Cent}_{\mathrm{Mat}_{r}(\mathrm{R})}\left(\mathrm{R} \otimes_{\mathrm{K}} \mathrm{K}_{\rho}\right)$. By Theorem 3.5.4 $\Gamma_{\Psi_{\rho}}(\mathrm{R})$ is equal to $\operatorname{Cent}_{\mathrm{GL}_{r}(\mathrm{R})}\left(\mathrm{R} \otimes_{\mathrm{K}} \mathrm{K}_{\rho}\right)$, which is the group of units in $\operatorname{Cent}_{\mathrm{Mat}_{r}(\mathrm{R})}\left(\mathrm{R} \otimes_{\mathrm{K}} \mathrm{K}_{\rho}\right)$. It follows that $\mathrm{R} \otimes_{\mathrm{k}} W_{i}$ is a $\Gamma_{\Psi_{\rho}}(\mathrm{R})$-module, and hence $W_{i} \in \boldsymbol{\operatorname { R e p }}\left(\Gamma_{M_{\rho}}, \mathrm{k}\right)$.

\section{Drinfeld LOGarithms And $\operatorname{Ext}_{\mathcal{T}}^{1}\left(\mathbf{1}, M_{\rho}\right)$}

In this section, we fix a Drinfeld A-module $\rho$ of rank $r$ defined over $\bar{k}$ as in (3.1.1) with $\kappa_{r}=1$, and we fix an $A$-basis $\left\{\omega_{1}, \ldots, \omega_{r}\right\}$ of $\Lambda_{\rho}$. We let $M_{\rho}$ be the $t$-motive associated to $\rho$ together with a fixed $\bar{k}(t)$-basis $\mathbf{m} \in \operatorname{Mat}_{r \times 1}\left(M_{\rho}\right), \Phi_{\rho}$ as in (3.3.1), and $\Psi_{\rho}$ as in (3.4.6). Finally, we let $K_{\rho}:=k \otimes_{A} \operatorname{End}(\rho)$ and $\mathrm{K}_{\rho}:=\operatorname{End}_{\mathcal{T}}\left(M_{\rho}\right)$.

\subsection{Endomorphisms of $t$-motives.}

Proposition 4.1.1. Given $e \in \operatorname{End}_{\mathcal{T}}\left(M_{\rho}\right)$, let $E=\left(E_{i j}\right) \in \operatorname{Mat}_{r}(\bar{k}(t))$ satisfy $e(\mathbf{m})=E \mathbf{m}$.

(a) Each entry $E_{i j}$ is regular at $t=\theta, \theta^{q}, \theta^{q^{2}}, \ldots$

(b) $E_{21}(\theta)=\cdots=E_{r 1}(\theta)=0$.

(c) $E_{11}(\theta)$ lies in $K_{\rho}$.

Proof. In [17, p. 146], it has been shown that the denominator of each $E_{i j}$ is in A, proving (a). To prove (b), define $\eta:=\Psi_{\rho}^{-1} E \Psi_{\rho}$. Using the equation $\Phi_{\rho} E=$ $E^{(-1)} \Phi_{\rho}$, we have $\eta^{(-1)}=\eta$ and hence $\eta \in \operatorname{Mat}_{r}(\mathrm{k})$. Using (3.4.6) and (3.4.8), specializing at $t=\theta$ on both sides of $\eta \Upsilon^{(1)} V=\Upsilon^{(1)} V E$ gives rise to the following relation:

$$
\begin{aligned}
-\sum_{i=1}^{r} \eta_{1 i}(\theta) \omega_{i}= & -E_{11}(\theta) \omega_{1}+\left(E_{21}(\theta) \kappa_{2}^{(-1)}+\cdots+E_{r 1}(\theta)\right) F_{\tau}\left(\omega_{1}\right) \\
& +\left(E_{21}(\theta) \kappa_{3}^{(-1)}+\cdots+E_{(r-1) 1}(\theta)\right) F_{\tau^{2}}\left(\omega_{1}\right) \\
& \vdots \\
& +\left(E_{21}(\theta) \kappa_{r-1}^{(-1)}+E_{31}(\theta)\right) F_{\tau^{r-2}}\left(\omega_{1}\right) \\
& +E_{21}(\theta) F_{\tau^{r-1}}\left(\omega_{1}\right) .
\end{aligned}
$$

Let $s=\left[K_{\rho}: k\right]$. By Theorem 3.5.4 (or by [4, Prop. 2]), we see that $\left\{F_{\tau}\left(\omega_{1}\right), \ldots\right.$, $\left.F_{\tau^{r-1}}\left(\omega_{1}\right)\right\}$ joined with any maximal $K_{\rho}$-linearly independent subset of $\Lambda_{\rho}$ are themselves linearly independent over $\bar{k}$. It follows that for each $1 \leq i \leq r-1$ the coefficients of $F_{\tau^{i}}\left(\omega_{1}\right)$ in (4.1.2) are zero. Thus we have $E_{21}(\theta)=\cdots=E_{r 1}(\theta)=0$. To 
prove (c), specialize both sides of the first columns of $\eta \Upsilon^{(1)} V=\Upsilon^{(1)} V E$ at $t=\theta$. Then by (b),

$$
\eta(\theta)\left(\begin{array}{c}
-\omega_{1} \\
\vdots \\
-\omega_{r}
\end{array}\right)=E_{11}(\theta)\left(\begin{array}{c}
-\omega_{1} \\
\vdots \\
-\omega_{r}
\end{array}\right) .
$$

Since $\eta(\theta) \in \operatorname{Mat}_{r}(k)$, the definition of $\operatorname{End}(\rho)$ implies that $E_{11}(\theta)$ falls in $K_{\rho}$.

4.2. The $\mathrm{K}_{\rho}$-independence of $X_{i}$ in $\operatorname{Ext}_{\mathcal{T}}^{1}\left(\mathbf{1}, M_{\rho}\right)$. Given any $u \in \mathbb{C}_{\infty}$ with $\exp _{\rho}(u)=\alpha \in \bar{k}$, we set $f_{u}$ to be the Anderson generating function of $u$ as in (3.4.1). Let $\mathbf{h}_{\alpha}$ be the column vector $(\alpha, 0, \ldots, 0)^{\operatorname{tr}} \in \operatorname{Mat}_{r \times 1}(\bar{k})$. We define the pre-t-motive $X_{\alpha}$ of dimension $r+1$ over $\bar{k}(t)$ on which multiplication by $\sigma$ is given by $\Phi_{\alpha}:=\left(\begin{array}{ll}\Phi_{\rho} & \mathbf{0} \\ \mathbf{h}_{\alpha}^{\text {tr }} & 1\end{array}\right)$. We further define

$$
\mathbf{g}_{\alpha}:=\left(\begin{array}{c}
-(t-\theta) f_{u}(t)-\alpha \\
-\left(\kappa_{2}^{(-1)} f_{u}^{(1)}(t)+\cdots+\kappa_{r-1}^{(-1)} f_{u}^{(r-2)}(t)+f_{u}^{(r-1)}(t)\right) \\
-\left(\kappa_{3}^{(-2)} f_{u}^{(1)}(t)+\cdots+\kappa_{r-1}^{(-2)} f_{u}^{(r-3)}(t)+f_{u}^{(r-2)}(t)\right) \\
\vdots \\
-f_{u}^{(1)}(t)
\end{array}\right) \in \operatorname{Mat}_{r \times 1}(\mathbb{T})
$$

and then we have the difference equation $\Phi_{\rho}^{\operatorname{tr}} \mathbf{g}_{\alpha}^{(-1)}=\mathbf{g}_{\alpha}+\mathbf{h}_{\alpha}$. Putting

$$
\Psi_{\alpha}:=\left(\begin{array}{cc}
\Psi_{\rho} & \mathbf{0} \\
\mathbf{g}_{\alpha}^{\text {tr }} \Psi_{\rho} & 1
\end{array}\right) \in \operatorname{Mat}_{r+1}(\mathbb{T})
$$

we see that $\Psi_{\alpha}^{(-1)}=\Phi_{\alpha} \Psi_{\alpha}$, and hence $X_{\alpha}$ is rigid analytically trivial. Based on Proposition 3.3.2, the argument in [17, Prop. 6.1.3] shows that $X_{\alpha}$ is a $t$-motive and so $X_{\alpha}$ represents a class in $\operatorname{Ext}_{\mathcal{T}}^{1}\left(\mathbf{1}, M_{\rho}\right)$.

The group $\operatorname{Ext}_{\mathcal{T}}^{1}\left(\mathbf{1}, M_{\rho}\right)$ also has the structure of a $\mathrm{K}_{\rho}$-vector space by pushing out along $M_{\rho}$. To see this explicitly, suppose the operation of $e \in \mathrm{K}_{\rho}$ on $M_{\rho}$ is represented by $E \in \operatorname{Mat}_{r}(\bar{k}(t))$ as in Proposition 4.1.1. By choosing a $\bar{k}(t)$-basis for an extension $X$ so that multiplication by $\sigma$ on $X$ is represented by $\left(\begin{array}{cc}\Phi_{\rho} & 0 \\ \mathbf{v} & 1\end{array}\right)$, we see that multiplication by $\sigma$ on the push-out $e_{*} X$ is represented by $\left(\begin{array}{cc}\Phi_{\rho} & 0 \\ \mathbf{v} E & 1\end{array}\right)$. Likewise, as is also standard, the Baer sum in $\operatorname{Ext}_{\mathcal{T}}^{1}\left(\mathbf{1}, M_{\rho}\right)$ is achieved by adding entries in the $\mathbf{v}$ row vector.

Theorem 4.2.2. Suppose $u_{1}, \ldots, u_{n} \in \mathbb{C}_{\infty}$ satisfy $\exp _{\rho}\left(u_{i}\right)=\alpha_{i} \in \bar{k}$ for $i=$ $1, \ldots, n$. For each $i$, let $X_{i}:=X_{\alpha_{i}}$ be as above. If $\operatorname{dim}_{K_{\rho}} \operatorname{Span}_{K_{\rho}}\left(\omega_{1}, \ldots, \omega_{r}, u_{1}\right.$, $\left.\ldots, u_{n}\right)=r / s+n$, then the classes of $X_{1}, \ldots, X_{n}$ in $\operatorname{Ext}_{\mathcal{T}}^{1}\left(\mathbf{1}, M_{\rho}\right)$ are linearly independent over $\mathrm{K}_{\rho}$.

Proof. For each $i$ we define $\mathbf{h}_{i}:=\mathbf{h}_{\alpha_{i}}, \mathbf{g}_{i}:=\mathbf{g}_{\alpha_{i}}, \Phi_{i}:=\Phi_{\alpha_{i}}, \Psi_{i}:=\Psi_{\alpha_{i}}$ as in the preceding paragraphs. Suppose that there exist $e_{1}, \ldots, e_{n} \in \mathrm{K}_{\rho}$, not all zero, so that $N:=e_{1 *} X_{1}+\cdots+e_{n_{*}} X_{n}$ is trivial in $\operatorname{Ext}_{\mathcal{T}}^{1}\left(\mathbf{1}, M_{\rho}\right)$. Fix $E_{i} \in \operatorname{Mat}_{r}(\bar{k}(t))$ so that $e_{i}(\mathbf{m})=E_{i} \mathbf{m}$ for each $i$. By choosing an appropriate $\bar{k}(t)$-basis $\mathbf{n}$ for $N$, multiplication by $\sigma$ on $N$ and a corresponding rigid analytic trivialization are represented by the matrices

$\Phi_{N}:=\left(\begin{array}{cc}\Phi_{\rho} & \mathbf{0} \\ \sum_{i=1}^{n} \mathbf{h}_{i}^{\operatorname{tr}} E_{i} & 1\end{array}\right) \in \mathrm{GL}_{r+1}(\bar{k}(t)), \Psi_{N}:=\left(\begin{array}{cc}\Psi_{\rho} & \mathbf{0} \\ \sum_{i=1}^{n} \mathbf{g}_{i}^{\operatorname{tr}} E_{i} \Psi_{\rho} & 1\end{array}\right) \in \mathrm{GL}_{r+1}(\mathbb{L})$. 
Since $N$ is trivial in $\operatorname{Ext}_{\mathcal{T}}^{1}\left(\mathbf{1}, M_{\rho}\right)$, there exists a matrix

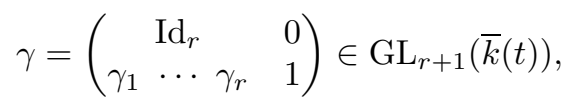

so that if we set $\mathbf{n}^{\prime}:=\gamma \mathbf{n}$, then we have $\sigma \mathbf{n}^{\prime}=\left(\Phi_{\rho} \oplus(1)\right) \mathbf{n}^{\prime}$, where $\Phi_{\rho} \oplus(1) \in$ $\mathrm{GL}_{r+1}(\bar{k}(t))$ is the block diagonal matrix with $\Phi_{\rho}$ and 1 down the diagonal and zeros elsewhere. Thus,

$$
\gamma^{(-1)} \Phi_{N}=\left(\Phi_{\rho} \oplus(1)\right) \gamma
$$

Note that by [17, p. 146] all denominators of $\gamma$ are in $\mathbb{F}_{q}[t]$ and so in particular each $\gamma_{i}$ is regular at $t=\theta, \theta^{q}, \theta^{q^{2}}, \ldots$

Using (4.2.3), we have $\left(\gamma \Psi_{N}\right)^{(-1)}=\left(\Phi_{\rho} \oplus(1)\right)\left(\gamma \Psi_{N}\right)$. By [17, §4.1.6], for some $\delta=\left(\begin{array}{ccc}\operatorname{cd}_{1} & \cdots & 0 \\ \delta_{1} & \delta_{r} & 1\end{array}\right) \in \mathrm{GL}_{r+1}(\mathrm{k})$, we have $\gamma \Psi_{N}=\left(\Psi_{\rho} \oplus(1)\right) \delta$. It follows that $\left(\gamma_{1}, \ldots, \gamma_{r}\right)+\sum_{i=1}^{n} \mathbf{g}_{i}^{\operatorname{tr}} E_{i}=\left(\delta_{1}, \ldots, \delta_{r}\right) \Psi_{\rho}^{-1}$. Note that for each $i$, the first entry of $\mathrm{g}_{i}(\theta)$ is given by $u_{i}-\alpha_{i}$. Hence, using Proposition 4.1.1(b) and specializing both sides of this equation at $t=\theta$, we obtain

$$
\gamma_{1}(\theta)+\sum_{i=1}^{n}\left(u_{i}-\alpha_{i}\right)\left(E_{i}\right)_{11}(\theta)=-\sum_{j=1}^{r} \delta_{j}(\theta) \omega_{j} .
$$

On the other hand, we find $\gamma_{n}^{(-1)}(t-\theta)+\sum_{i=1}^{n} \alpha_{i}\left(E_{i}\right)_{11}=\gamma_{1}$ from the $(r+1,1)$ entry of both sides of (4.2.3). Specializing both sides of this equation at $t=\theta$ implies that $\gamma_{1}(\theta)=\sum_{i=1}^{n} \alpha_{i}\left(E_{i}\right)_{11}(\theta)$. Thus, from (4.2.4) we obtain

$$
\sum_{i=1}^{n}\left(E_{i}\right)_{11}(\theta) u_{i}+\sum_{j=1}^{r} \delta_{j}(\theta) \omega_{j}=0
$$

The assumption that $e_{1}, \ldots, e_{n}$ are not all zero implies that $E_{i}$ is nonzero for some $i$. By Proposition 3.3 .2 we have $\mathrm{K}_{\rho} \cong K_{\rho}$ and $E_{i}$ is invertible, and hence Proposition 4.1.1(b,c) implies that $\left(E_{i}\right)_{11}(\theta) \in K_{\rho}^{\times}$, which gives the desired contradiction.

\section{Algebraic independence of Drinfeld logarithmS}

5.1. The case of $A=\mathbb{F}_{q}[t]$. We continue with the notation of 4 . We suppose we have $u_{1}, \ldots, u_{n} \in \mathbb{C}_{\infty}$ with $\exp _{\rho}\left(u_{i}\right)=\alpha_{i} \in \bar{k}$ for each $i$, and then maintain the choices from Theorem 4.2 .2 and its proof. We note that the rigid analytic trivializations $\Psi_{i}$ of each $X_{i}$ have entire entries, which is assured by [2, Prop. 3.1.3]. The matrix $\Psi:=\bigoplus_{i=1}^{n} \Psi_{i}$ provides a rigid analytic trivialization for the $t$-motive $X:=\bigoplus_{i=1}^{n} X_{i}$. Our goal in this section is to calculate the Galois group $\Gamma_{X}$. From the construction of each $\Psi_{i}$, we see that

$$
\bar{k}(\Psi(\theta))=\bar{k}\left(\bigcup_{i=1}^{r-1} \bigcup_{m=1}^{n} \bigcup_{j=1}^{r}\left\{\omega_{j}, F_{\tau^{i}}\left(\omega_{j}\right), u_{m}, F_{\tau^{i}}\left(u_{m}\right)\right\}\right) .
$$

By Theorem 2.3.3 we have $\operatorname{dim} \Gamma_{\Psi}=\operatorname{tr} \cdot \operatorname{deg}_{\bar{k}} \bar{k}(\Psi(\theta))$, and Theorem 3.5.4 then implies that

$$
\operatorname{dim} \Gamma_{\Psi}=\operatorname{tr} . \operatorname{deg}_{\bar{k}} \bar{k}(\Psi(\theta)) \leq \frac{r^{2}}{s}+r n .
$$


Now let $N$ be the $t$-motive defined by $\Phi_{N} \in \mathrm{GL}_{r n+1}(\bar{k}(t))$ with rigid analytic trivialization $\Psi_{N} \in \mathrm{GL}_{r n+1}(\mathbb{T})$ :

$$
\Phi_{N}:=\left(\begin{array}{cccc}
\Phi_{\rho} & & & \\
& \ddots & & \\
& & \Phi_{\rho} & \\
\mathbf{h}_{\alpha_{1}}^{\mathrm{tr}} & \cdots & \mathbf{h}_{\alpha_{n}}^{\operatorname{tr}} & 1
\end{array}\right), \quad \Psi_{N}:=\left(\begin{array}{cccc}
\Psi_{\rho} & & \\
& \ddots & & \\
& & \Psi_{\rho} & \\
\mathbf{g}_{\alpha_{1}}^{\operatorname{tr}} \Psi_{\rho} & \cdots & \mathbf{g}_{\alpha_{n}}^{\operatorname{tr}} \Psi_{\rho} & 1
\end{array}\right) .
$$

By the definition of $\Gamma_{\Psi_{N}}$, for any k-algebra $\mathrm{R}$ each element $\nu \in \Gamma_{\Psi_{N}}(\mathrm{R})$ is of the form $\left(\begin{array}{c}\oplus_{i=1}^{n} \gamma \\ *\end{array}\right)$, for some $\gamma \in \Gamma_{\Psi_{\rho}}(\mathrm{R})$. Note that $N$ is an extension of $\mathbf{1}$ by $M_{\rho}^{n}$, which is the pullback of $X \rightarrow \mathbf{1}^{n}$ and the diagonal embedding $\mathbf{1} \hookrightarrow \mathbf{1}^{n}$. Thus, as the two $t$-motives $X$ and $N$ generate the same Tannakian subcategory of $\mathcal{T}$, the Galois groups $\Gamma_{X}$ and $\Gamma_{N}$ are isomorphic, in particular $\Gamma_{\Psi} \cong \Gamma_{N}$. Note that by Theorem 2.3.1 any element $\nu$ in $\Gamma_{N}$ is of the form

$$
\left(\begin{array}{cccc}
\gamma & & & \\
& \ddots & & \\
& & \gamma & \\
\mathbf{v}_{1} & \cdots & \mathbf{v}_{n} & 1
\end{array}\right)
$$

for some $\gamma \in \Gamma_{M_{\rho}}$ and some $\mathbf{v}_{1}, \ldots, \mathbf{v}_{n} \in \mathbb{G}_{\mathrm{a}}{ }^{r}$. As $M_{\rho}^{n}$ is a sub-t-motive of $N$, we have the short exact sequence of affine algebraic group schemes over $k$,

$$
1 \rightarrow W \rightarrow \Gamma_{N} \stackrel{\pi}{\rightarrow} \Gamma_{M_{\rho}} \rightarrow 1
$$

where the surjective map $\pi: \Gamma_{N} \rightarrow \Gamma_{M_{\rho}}$ is the projection map given by $\nu \mapsto \gamma$ (cf. [7, p.22]). We notice that (5.1.2) gives rise to an action of any $\gamma \in \Gamma_{M_{\rho}}$ on

$$
\mathbf{v}=\left(\begin{array}{cccc}
\operatorname{Id}_{r} & & & \\
& \ddots & & \\
& & \operatorname{Id}_{r} & \\
\mathbf{v}_{1} & \cdots & \mathbf{v}_{n} & 1
\end{array}\right) \in W
$$

given by

$$
\left(\begin{array}{cccc}
\operatorname{Id}_{r} & & & \\
& \ddots & & \\
& & \operatorname{Id}_{r} & \\
\mathbf{v}_{1} \gamma^{-1} & \cdots & \mathbf{v}_{n} \gamma^{-1} & 1
\end{array}\right)
$$

In what follows, we will show that $W$ can be identified with a $\Gamma_{M_{\rho}}$-submodule of $\left(M_{\rho}^{n}\right)^{\mathrm{B}} \simeq\left(M_{\rho}^{\mathrm{B}}\right)^{n}$. Let $\mathbf{n} \in \operatorname{Mat}_{(r n+1) \times 1}(N)$ be the $\bar{k}(t)$-basis of $N$ such that $\sigma \mathbf{n}=\Phi_{N} \mathbf{n}$. We write $\mathbf{n}=\left(\mathbf{n}_{1}, \ldots, \mathbf{n}_{n}, x\right)^{\mathrm{tr}}$, where $\mathbf{n}_{i} \in \operatorname{Mat}_{r \times 1}(N)$. Notice that $\left(\mathbf{n}_{1}, \ldots, \mathbf{n}_{n}\right)^{\operatorname{tr}}$ is a $\bar{k}(t)$-basis of $M_{\rho}^{n}$. Recall that the entries of $\Psi_{N}^{-1} \mathbf{n}$ form a k-basis of $N^{\mathrm{B}}$, and $\mathbf{u}:=\left(\Psi_{\rho}^{-1} \mathbf{n}_{1}, \ldots, \Psi_{\rho}^{-1} \mathbf{n}_{n}\right)^{\text {tr }}$ is a k-basis of $M_{\rho}^{n}$. Given any k-algebra $\mathrm{R}$, we recall the action of $\Gamma_{M_{\rho}}(\mathrm{R})$ on $\mathrm{R} \otimes_{\mathrm{k}}\left(M_{\rho}^{\mathrm{B}}\right)^{n}$ as follows (cf. [17, $\S 4.5])$ : for any $\gamma \in \Gamma_{M_{\rho}}(\mathrm{R})$ and any $\mathbf{v}_{i} \in \operatorname{Mat}_{1 \times r}(\mathrm{R}), 1 \leq i \leq n$, the action of $\gamma$ on $\left(\mathbf{v}_{1}, \ldots, \mathbf{v}_{n}\right) \cdot \mathbf{u} \in \mathrm{R} \otimes_{\mathrm{k}}\left(M_{\rho}^{\mathrm{B}}\right)^{n}$ is presented as

$$
\left(\mathbf{v}_{1}, \ldots, \mathbf{v}_{n}\right) \cdot \mathbf{u} \mapsto\left(\mathbf{v}_{1} \gamma^{-1}, \ldots, \mathbf{v}_{n} \gamma^{-1}\right) \cdot \mathbf{u} .
$$

It follows that when a basis $\mathbf{u}$ of $\left(M_{\rho}^{\mathrm{B}}\right)^{n}$ is fixed as above, the action of $\Gamma_{M_{\rho}}$ on $\left(M_{\rho}^{\mathrm{B}}\right)^{n}$ is compatible with the action of $\Gamma_{M_{\rho}}$ on $W$ described in the previous 
paragraph. Regarding $\left(M_{\rho}^{\mathrm{B}}\right)^{n}$ as a vector group over $\mathrm{k}$, it follows that $\Gamma_{N}$ is a subgroup of the group scheme $\Gamma_{M_{\rho}} \ltimes\left(M_{\rho}^{\mathrm{B}}\right)^{n}$ over $k$, which satisfies the short exact sequence

$$
1 \rightarrow\left(M_{\rho}^{\mathrm{B}}\right)^{n} \rightarrow \Gamma_{M_{\rho}} \ltimes\left(M_{\rho}^{\mathrm{B}}\right)^{n} \rightarrow \Gamma_{M_{\rho}} \rightarrow 1 .
$$

We also see that $W$ is equal to the scheme-theoretic intersection $\Gamma_{N} \cap\left(M_{\rho}^{\mathrm{B}}\right)^{n}$.

Lemma 5.1.3. The k-group scheme $W$ is the k-vector subgroup of $\left(M_{\rho}^{\mathrm{B}}\right)^{n}$ arising from a $\Gamma_{M_{\rho}}$-submodule. In particular, it is k-smooth.

Proof. Recall that $\mathrm{K}_{\rho}:=\operatorname{End}_{\mathcal{T}}\left(M_{\rho}\right)$ is naturally embedded into $\operatorname{End}\left(M_{\rho}^{B}\right)$, so we can regard $M_{\rho}^{\mathrm{B}}$ as a vector space $\mathscr{V}^{\prime}$ over $\mathrm{K}_{\rho}$. Let $G^{\prime}$ denote the $\mathrm{K}_{\rho}$-group $\mathrm{GL}\left(\mathscr{V}^{\prime}\right)$. Corollary 3.5.6 says that $\Gamma_{M_{\rho}}$ naturally coincides with the Weil restriction of scalars $\mathrm{R}_{\mathrm{K}_{\rho} / \mathrm{k}}\left(G^{\prime}\right)$ over k. Let $V^{\prime}$ be the $\mathrm{K}_{\rho}$-vector space $\mathscr{V}^{\prime \oplus n}$, and let $V$ be the vector group over $k$ associated to the underlying k-vector space. By Theorem 2.3.1(b), $\Gamma_{N}$ is smooth. Hence, our situation is a special case of Example A.1.2 of Appendix A (by taking $G=\Gamma_{M_{\rho}}, \Gamma=\Gamma_{N}, k^{\prime}=\mathrm{K}_{\rho}$, and $k=\mathrm{k}$ there), so Proposition A.1.3 provides the desired result.

Remark 5.1.4. In the case that $K_{\rho}$ is separable over $k$, one can prove Lemma 5.1 .3 by showing that the induced tangent map $d \pi$ is surjective onto the Lie algebra of $\Gamma_{M_{\rho}}$ along the lines of the arguments of the proof of [7, Prop. 4.1.2].

Theorem 5.1.5. Given $u_{1}, \ldots, u_{n} \in \mathbb{C}_{\infty}$ with $\exp _{\rho}\left(u_{i}\right)=\alpha_{i} \in \bar{k}$ for $i=1, \ldots, n$, we let $X_{1}, \ldots, X_{n} \in \operatorname{Ext}_{\mathcal{T}}^{1}\left(1, M_{\rho}\right), N$ and $\Psi$ be defined as above. Suppose that $\omega_{1}, \ldots, \omega_{r / s}, u_{1}, \ldots, u_{n}$ are linearly independent over $K_{\rho}$. Then $\Gamma_{\Psi}$ is an extension of $\Gamma_{M_{\rho}}$ by a vector group of dimension $r n$ defined over $\mathrm{k}$, and so $\operatorname{dim} \Gamma_{\Psi}=r(\mathrm{r} / \mathrm{s}+$ n). In particular, by (5.1.1),

$$
\bigcup_{i=1}^{r-1} \bigcup_{m=1}^{n} \bigcup_{j=1}^{r / s}\left\{\omega_{j}, F_{\tau^{i}}\left(\omega_{j}\right), u_{m}, F_{\tau^{i}}\left(u_{m}\right)\right\}
$$

is an algebraically independent set over $\bar{k}$.

Proof. By (5.1.1), our task is to prove that $\operatorname{dim} \Gamma_{\Psi}=r(r / s+n)$, which is equivalent to proving that $\operatorname{dim} W=r n$ by Theorem 3.5.4 and (5.1.2). As Lemma 5.1.3 implies that $W$ is a $\Gamma_{M_{\rho}}$-submodule of $\left(M_{\rho}^{n}\right)^{\mathrm{B}}$, by the equivalence of the categories, $\mathcal{T}_{M_{\rho}} \approx$ $\boldsymbol{\operatorname { R e p }}\left(\Gamma_{M_{\rho}}, \mathrm{k}\right)$, there exists a sub-t-motive $U$ of $M_{\rho}^{n}$ so that $W \cong U^{\mathrm{B}}$. Therefore, to prove $\operatorname{dim} W=r n$ it suffices to prove that $U^{\mathrm{B}}=\left(M_{\rho}^{n}\right)^{\mathrm{B}}$.

We claim that $N / U$ is split as a direct sum of $M_{\rho}^{n} / U$ and 1 . To prove this, we follow an argument of Hardouin (see 15, Lem. 2.3]). Since $W \cong U^{\mathrm{B}}, \Gamma_{N}$ acts on $N^{\mathrm{B}} / U^{\mathrm{B}}$ through the quotient $\Gamma_{N} / W \cong \Gamma_{M_{\rho}}$ via (5.1.2). It follows that $N^{\mathrm{B}} / U^{\mathrm{B}}$ is an extension of $\mathrm{k}$ by $\left(M_{\rho}^{n}\right)^{\mathrm{B}} / U^{\mathrm{B}}$ in the category $\operatorname{Rep}\left(\Gamma_{M_{\rho}}, \mathrm{k}\right)$. By the equivalence $\mathcal{T}_{M_{\rho}} \approx \boldsymbol{R e p}\left(\Gamma_{M_{\rho}}, \mathrm{k}\right)$ and Corollary 3.5.7 we see that the extension $N / U$ is trivial in $\operatorname{Ext}_{\mathcal{T}}^{1}\left(\mathbf{1}, M_{\rho}^{n} / U\right)$.

Now suppose on the contrary that $U^{\mathrm{B}} \subsetneq\left(M_{\rho}^{n}\right)^{\mathrm{B}}$. By Corollary 3.3 .3 we have that $M_{\rho}^{n}$ is completely reducible in $\mathcal{T}_{M_{\rho}}$. As $U$ is a proper sub-t-motive of $M_{\rho}^{n}$, there exists a nontrivial morphism $\phi \in \operatorname{Hom}_{\mathcal{T}}\left(M_{\rho}^{n}, M_{\rho}\right)$ so that $U \subseteq \operatorname{Ker} \phi$. Moreover, 
the morphism $\phi$ factors through the map $M_{\rho}^{n} / U \rightarrow M_{\rho}^{n} / \operatorname{Ker} \phi$ :

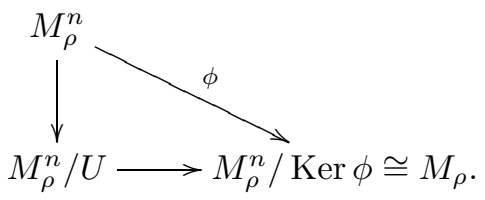

Since $\phi \in \operatorname{Hom}_{\mathcal{T}}\left(M_{\rho}^{n}, M_{\rho}\right)$, we can write $\phi\left(m_{1}, \ldots, m_{n}\right)=\sum_{i=1}^{n} e_{i}\left(m_{i}\right)$ for some $e_{1}, \ldots, e_{n} \in \mathrm{K}_{\rho}$, not all zero. Then the pushout $\phi_{*} N=e_{1 *} X_{1}+\cdots+e_{n *} X_{n}$ is a quotient of $N / U$. By the claim above, it follows that $\phi_{*} N$ is trivial in $\operatorname{Ext}_{\mathcal{T}}^{1}\left(1, M_{\rho}\right)$. But by Theorem 4.2.2 this contradicts the $\mathrm{K}_{\rho}$-linear independence of $X_{1}, \ldots, X_{n}$ in $\operatorname{Ext}_{\mathcal{T}}^{1}\left(\mathbf{1}, M_{\rho}\right)$.

Corollary 5.1.6. Let $\rho$ be a Drinfeld A-module of rank $r$ defined over $\bar{k}$. Let $u_{1}, \ldots, u_{n} \in \mathbb{C}_{\infty}$ satisfy $\exp _{\rho}\left(u_{i}\right) \in \bar{k}$ for $i=1, \ldots, n$, and suppose that they are linearly independent over $K_{\rho}$. Let $\delta_{1}, \ldots, \delta_{r}$ be a basis of $H_{\mathrm{DR}}(\rho)$ defined over $\bar{k}$. Then the rn quantities

$$
\bigcup_{j=1}^{r}\left\{F_{\delta_{j}}\left(u_{1}\right), \ldots, F_{\delta_{j}}\left(u_{n}\right)\right\}
$$

are algebraically independent over $\bar{k}$.

Proof. Every Drinfeld A-module defined over $\bar{k}$ is isomorphic over $\bar{k}$ to one for which the leading coefficient of $\rho_{t}$ is 1 . It is a routine matter to check that the desired result is invariant under isomorphism, so we will assume without loss of generality that this leading coefficient is 1 . Define the $K_{\rho}$-vector space, $V:=$ $\operatorname{Span}_{K_{\rho}}\left(\omega_{1}, \ldots, \omega_{r}, u_{1}, \ldots, u_{n}\right)$, and let $\left\{v_{1}, \ldots, v_{\ell}\right\}$ be a $K_{\rho}$-basis of $V$. Certainly $r / s \leq \ell \leq r / s+n$. Since quasi-periodic functions $F_{\delta}(z)$ are linear in $\delta$, using (3.1.2) we have

$$
\bar{k}\left(\bigcup_{i=1}^{r-1} \bigcup_{m=1}^{n} \bigcup_{j=1}^{r}\left\{\omega_{j}, F_{\tau^{i}}\left(\omega_{j}\right), u_{m}, F_{\tau^{i}}\left(u_{m}\right)\right\}\right)=\bar{k}\left(\bigcup_{j=1}^{r}\left\{F_{\delta_{j}}\left(v_{1}\right), \ldots, F_{\delta_{j}}\left(v_{\ell}\right)\right\}\right) .
$$

By swapping out basis elements of $V$ as necessary (see the proof of [7, Thm. 4.3.3]), the result follows from Theorem 5.1.5.

5.2. The case of general A. In this section, we prove Theorems 1.1.1 1.2.2, and 1.2.3. Essentially they follow from Theorem 3.5.4 and Corollary 5.1.6 when we consider $\rho$ to be a Drinfeld $\mathbb{F}_{q}[t]$-module with complex multiplication.

We resume the notation from the introduction. However, in order to separate the roles of " $\mathrm{A}$ as operators" from " $A$ as scalars", we let $\mathrm{A}$ be the ring of functions on $\mathrm{X}$ that are regular away from $\infty$ with fraction field $\mathrm{k}$, and we let $A$ be a copy of $A$ with fraction field $k$ that serve as scalars. The fields $k_{\infty}$ and $\mathbb{C}_{\infty}$ are then extensions of $k$. Thus we follow the "two $t$ 's" convention of [14, §5.4].

Let $\rho$ be a rank $r$ Drinfeld $A$-module defined over $\bar{k}$. Let $\mathrm{R}$ be the endomorphism ring of $\rho$, considered to be an extension of $A$, and let $K_{\rho}$ be the fraction field of $R$. We fix a nonconstant element $t \in A$, and consider $\rho$ to be a Drinfeld $\mathbb{F}_{q}[t]$-module with complex multiplication by $\mathrm{R}$ and defined over $\bar{k}$. Note that the exponential function $\exp _{\rho}(z)$ and its period lattice remain unchanged when switching from $A$ to $\mathbb{F}_{q}[t]$. 
As for scalars, we let $\theta \in \bar{k}$ be chosen so that the extension $K_{\rho} / \mathbb{F}_{q}(\theta)$ is canonically isomorphic to $\mathrm{K}_{\rho} / \mathbb{F}_{q}(t)$, where as usual $K_{\rho} \subseteq \bar{k}$ is the fraction field of $\operatorname{End}(\rho)$. In this way $\bar{k}=\overline{\mathbb{F}_{q}(\theta)}$ and $\overline{k_{\infty}}=\overline{\mathbb{F}_{q}((1 / \theta))}$.

Proof of Theorem 1.1.1. Since $u_{1}, \ldots, u_{n}$ are assumed to be linearly independent over $K_{\rho}$, Corollary [5.1.6 dictates that, for any biderivation $\delta: \mathbb{F}_{q}[t] \rightarrow \bar{k}[\tau] \tau$, $F_{\delta}\left(u_{1}\right), \ldots, F_{\delta}\left(u_{n}\right)$ are algebraically independent over $\bar{k}$. In particular, when we take $\delta=\delta^{(1)}$, we know that $F_{\delta^{(1)}}(z)=z-\exp _{\rho}(z)$, and since $\exp _{\rho}\left(u_{i}\right) \in \bar{k}$ for all $i$, the theorem is proved.

For the relevant background on the de Rham theory of Drinfeld A-modules, we refer the reader to [13, 26]. In order to distinguish the roles of $\rho$ over different base rings, we denote by $D\left(\rho, \mathbb{F}_{q}[t]\right)($ resp. $D(\rho, A))$ and $H_{\mathrm{DR}}\left(\rho, \mathbb{F}_{q}[t]\right)\left(\right.$ resp. $\left.H_{\mathrm{DR}}(\rho, \mathrm{A})\right)$ the spaces in $\$ 3.1$ when we regard $\rho$ as a Drinfeld $\mathbb{F}_{q}[t]$-module (resp. Drinfeld $A$ module). By the de Rham isomorphism (1.2.1) the restriction map $\left.\delta \mapsto \delta\right|_{\mathbb{F}_{q}[t]}$ : $D(\rho, \mathcal{A}) \rightarrow D\left(\rho, \mathbb{F}_{q}[t]\right)$ induces an injection

$$
H_{\mathrm{DR}}(\rho, A) \hookrightarrow H_{\mathrm{DR}}\left(\rho, \mathbb{F}_{q}[t]\right) .
$$

Moreover, it is straightforward to check that $F_{\delta}(z)=F_{\left.\delta\right|_{\mathbb{q}_{q}[t]}}(z)$ for all $\delta \in D(\rho, A)$.

Proofs of Theorems 1.2 .2 and 1.2.3. Let $s:=\left[\mathrm{K}_{\rho}: \mathrm{k}\right], \ell:=\left[\mathrm{k}: \mathbb{F}_{q}(t)\right]$. Then $\rho$ is a Drinfeld $\mathbb{F}_{q}[t]$-module of rank $r \ell$. Selecting a basis $\left\{\delta_{1}, \ldots, \delta_{r}\right\}$ for $H_{\mathrm{DR}}(\rho, A)$ defined over $\bar{k}$, we extend it to a basis $\left\{\eta_{1}:=\left.\delta_{1}\right|_{\mathbb{F}_{q}[t]}, \ldots, \eta_{r}:=\left.\delta_{r}\right|_{\mathbb{F}_{q}[t]}, \eta_{r+1}, \ldots, \eta_{r \ell}\right\}$ of $H_{\mathrm{DR}}\left(\rho, \mathbb{F}_{q}[t]\right)$ also defined over $\bar{k}$. We first prove Theorem 1.2.3, by applying Corollary 5.1 .6 to $\rho$ as a Drinfeld $\mathbb{F}_{q}[t]$-module. Suppose $u_{1}, \ldots, u_{n} \in \mathbb{C}_{\infty}$ satisfy $\exp _{\rho}\left(u_{i}\right) \in \bar{k}$ for each $i=1, \ldots, n$ and are linearly independent over $K_{\rho}$. Then the set

$$
\bigcup_{j=1}^{r \ell}\left\{F_{\eta_{j}}\left(u_{1}\right), \ldots, F_{\eta_{j}}\left(u_{n}\right)\right\}
$$

is algebraically independent over $\bar{k}$, whence so is the subset $\cup_{i=1}^{n} \cup_{j=1}^{r}\left\{F_{\delta_{j}}\left(u_{i}\right)\right\}$.

By these same considerations, Theorem 1.2.2 is now a special case of Theorem 1.2.3. If we select periods $\omega_{1}, \ldots, \omega_{r / s} \in \Lambda_{\rho}$ that are linearly independent over $K_{\rho}$, the $r^{2} / s$ quantities

$$
\bigcup_{j=1}^{r}\left\{F_{\eta_{j}}\left(\omega_{1}\right), \ldots, F_{\eta_{j}}\left(\omega_{r / s}\right)\right\}
$$

are algebraically independent over $\bar{k}$, and so tr. $\operatorname{deg}_{\bar{k}} \bar{k}\left(\mathrm{P}_{\rho}\right)=r^{2} / s$.

\section{Appendix A. Subgroups of a SEMidirect Product}

A.1. Main result. Let $k$ be a field, $k^{\prime}$ a nonzero finite reduced $k$-algebra (i.e., $k^{\prime}=\prod k_{i}^{\prime}$ for finite extension fields $\left.k_{i}^{\prime} / k\right)$, and $G^{\prime}$ a reductive group over $\operatorname{Spec}\left(k^{\prime}\right)$ with connected fibers. We allow for the possibility that $k^{\prime}$ is not $k$-étale (i.e., some $k_{i}^{\prime}$ is not separable over $k$ ). Let $V$ be a commutative smooth connected unipotent $k$-group equipped with a left action by the Weil restriction $G=\mathrm{R}_{k^{\prime} / k}\left(G^{\prime}\right)$. By [8, Prop. A.5.2(4), Prop. A.5.9], the affine finite type $k$-group scheme $G$ is smooth and connected. Beware that if $k^{\prime}$ is not $k$-étale then $G$ is not reductive when $G^{\prime} \rightarrow \operatorname{Spec}\left(k^{\prime}\right)$ has a nontrivial fiber over some point in the non-étale locus of $k^{\prime}$ over $k$ [8, Ex. 1.6.1]. 
For a maximal $k$-torus $T$ in $G$, the scheme-theoretic fixed locus $V^{T}$ is smooth and connected (since the centralizer of $T$ in $G \ltimes V$ is smooth and connected yet equals $Z_{G}(T) \ltimes V^{T}$ ). We will be interested in cases where $V^{T}=0$ for some (equivalently, all) $T$.

Remark A.1.1. The reason we do not require $k^{\prime}$ to be a field in general is that in proofs it is useful to extend the ground field $k$ to a separable closure $k_{s}$, and $k_{s}^{\prime}:=k^{\prime} \otimes_{k} k_{s}$ is typically not a field. The $k_{s}$-algebra $k_{s}^{\prime}$ arises because for an affine $k^{\prime}$-scheme $X^{\prime}$ of finite type we have $\mathrm{R}_{k^{\prime} / k}\left(X^{\prime}\right)_{k_{s}}=\mathrm{R}_{k_{s}^{\prime} / k_{s}}\left(X_{k_{s}^{\prime}}^{\prime}\right)$.

Here is the situation that is of most interest to us.

Example A.1.2. Suppose $G^{\prime}$ is equipped with a linear representation $\rho^{\prime}$ on a finitely generated $k^{\prime}$-module $V^{\prime}$ such that $V^{\prime Z^{\prime}}=0$ for some central torus $Z^{\prime}$ in $G^{\prime}$. Also assume that the action of the Lie algebra $\mathfrak{z}^{\prime}$ on $V^{\prime}$ satisfies $V^{\mathfrak{z}^{\prime}}=0$ (equivalently, over every factor field of $k_{s}^{\prime}=k^{\prime} \otimes_{k} k_{s}$, each weight for the $Z^{\prime}$ action on $V^{\prime}$ is not divisible by $\operatorname{char}(k)$ in the geometric character lattice of the corresponding fiber of $\left.Z^{\prime}\right)$. For example, we could take $G^{\prime}=\mathrm{GL}\left(\mathscr{V}^{\prime}\right)$ for a finitely generated $k^{\prime}$-module $\mathscr{V}^{\prime}$ and $\rho^{\prime}$ to be the standard representation of $G^{\prime}$ on the direct $\operatorname{sum} V^{\prime}=\mathscr{V}^{\prime \oplus n}$ for any $n>0$.

Let $V$ be the vector group over $k$ underlying $V^{\prime}$, and equip it with its natural left action by $G:=\mathrm{R}_{k^{\prime} / k}\left(G^{\prime}\right)$ (acting via Weil restriction of $\rho^{\prime}$ ). The center of $G$ contains $\mathrm{R}_{k^{\prime} / k}\left(Z^{\prime}\right)$, so the maximal $k$-torus $Z$ in $\mathrm{R}_{k^{\prime} / k}\left(Z^{\prime}\right)$ is contained in every maximal $k$-torus $T$ of $G$. It is easy to check that the weights for the action of $Z_{k_{s}}$ on $V_{k_{s}}$ are all nontrivial, so $V^{Z}=0$ and hence $V^{T}=0$ for all $T$.

The main result of this appendix is:

Proposition A.1.3. With the notation and hypotheses as in Example A.1.2, let $\Gamma \subset G \ltimes V$ be a smooth closed $k$-subgroup such that $\Gamma \rightarrow G$ is surjective. The scheme-theoretic intersection $\Gamma \cap V$ is equal to $W:=R_{k^{\prime} / k}\left(W^{\prime}\right)$ for a unique $k^{\prime}$ submodule $W^{\prime}$ of $V^{\prime}$ that is moreover $G^{\prime}$-stable, and there exists a unique $v \in$ $(V / W)(k)=V^{\prime} / W^{\prime}$ such that $\Gamma$ is the preimage under $G \ltimes V \rightarrow G \ltimes(V / W)$ of the $v$-conjugate of $G$ in $G \ltimes(V / W)$.

In particular, $\Gamma \cap V$ is smooth and $\Gamma$ is connected, and if $k^{\prime}$ is a field with $G^{\prime}$ acting irreducibly on $V^{\prime}$ over $k^{\prime}$, then either $\Gamma=G \ltimes V$ or $\Gamma$ is the v-conjugate of $G$ for a unique $v \in V(k)$.

Remark A.1.4. The description of $W$ in terms of a $k^{\prime}$-submodule $W^{\prime}$ of $V^{\prime}$ relies crucially on the hypothesis that $V^{\mathfrak{z}^{\prime}}=0$ in Example A.1.2 For example, if $\operatorname{char}(k)=p>0$ and $k^{\prime} / k$ is a purely inseparable extension of degree $p$ then consider $V^{\prime}=k^{\prime}$ equipped with the action of $Z^{\prime}=G^{\prime}=\mathrm{GL}_{1}$ via $t . x=t^{p} x$. Note that $V^{\prime z^{\prime}}=V^{\prime}$ even though $V^{\prime Z^{\prime}}=0$, and the canonical $k$-subgroup $W=\mathbf{G}_{\mathrm{a}}$ in $V=\mathrm{R}_{k^{\prime} / k}\left(\mathbf{G}_{\mathrm{a}}\right)$ is $G$-stable since $k^{\prime p} \subseteq k$. Clearly $\Gamma:=G \ltimes W$ satisfies $\Gamma \cap V=W$ scheme-theoretically, so $\Gamma$ violates the conclusion of Proposition A.1.3 concerning the structure of $W$.

A.2. Proof of Proposition A.1.3. In view of the uniqueness assertion for $v$ in Proposition A.1.3 by Galois descent we may and do assume $k=k_{s}$. The action of $G \ltimes V$ on the normal $k$-subgroup $V$ factors through the natural action of the quotient $G$ since $V$ is commutative. Since $\Gamma \rightarrow G$ is surjective and $\Gamma(k)$ is Zariski-dense in $\Gamma$ (as $k=k_{s}$ and $\Gamma$ is smooth), it follows that $\Gamma(k)$ has Zariski-dense image in $G$. 
Thus, a smooth closed $k$-subgroup of $V$ is $G$-stable provided that it is normalized by $\Gamma(k)$ inside of $G \ltimes V$. In particular, the Zariski closure $V_{0}$ of $\Gamma(k) \cap V(k)$ in $V$ is a smooth closed $k$-subgroup of $V$ that is stable under the $G$-action on $V$. If $\Gamma \cap V$ is going to be smooth then it must necessarily equal $V_{0}$, so this motivates our work with $V_{0}$ in what follows. Much later in the argument we will prove that $\Gamma \cap V=V_{0}$.

Any surjection between smooth connected affine $k$-groups that is equivariant for actions by a torus restricts to a surjection between centralizers for the torus actions, so $V^{Z} \rightarrow\left(V / V_{0}\right)^{Z}$ is surjective. Thus, $\left(V / V_{0}\right)^{Z}=0$ since we assume $V^{Z}=0$. Before we address the structure of $V_{0}$, we first verify the following lemma, which amounts to the uniqueness for $v$ once we know that $\Gamma \cap V$ is smooth.

Lemma A.2.1. There is at most one $v \in\left(V / V_{0}\right)(k)$ such that $\Gamma / V_{0}$ is the $v$ conjugate of $G$ inside of $G \ltimes\left(V / V_{0}\right)$.

Proof. For any $k$-algebra $A$ and $A$-valued points $g, h$ of $G$ and $v, w$ of $V / V_{0}$, we have

$$
(g, v)(h, w)(g, v)^{-1}=\left(g h, h^{-1} v+w\right)\left(g^{-1},-g v\right)=\left(g h g^{-1}, g h^{-1} v-g v+g w\right) .
$$

Setting $g=1$, for any $v \in\left(V / V_{0}\right)(k)$ we see that the $v$-conjugate of $(h, w)$ is $\left(h, h^{-1} v-v+w\right)$. Thus, the $v$-conjugate of $G$ in $G \ltimes\left(V / V_{0}\right)$ is the graph of the map $G \rightarrow V / V_{0}$ defined by $h \mapsto h^{-1} v-v$. Uniqueness of $v$ reduces to the property $\left(V / V_{0}\right)(k)^{G}=0$, and this holds because $\left(V / V_{0}\right)^{Z}=0$.

Suppose we could show that the image $\bar{\Gamma}$ of $\Gamma$ in $G \ltimes\left(V / V_{0}\right)$ contains a $\left(V / V_{0}\right)(k)$ conjugate of $G$. Since $V(k) \rightarrow\left(V / V_{0}\right)(k)$ is surjective (as $k=k_{s}$ and $V_{0}$ is smooth), such a containment would bring us to the situation (after a $V(k)$-conjugation on $\Gamma)$ that $\Gamma=G \ltimes(\Gamma \cap V)$, which forces the scheme-theoretic intersection $\Gamma \cap V$ to be smooth (as a direct factor of a smooth $k$-scheme is smooth) and therefore equal to $V_{0}$, so we would be done. Our problem is therefore reduced to establishing that $V_{0}=\mathrm{R}_{k^{\prime} / k}\left(W^{\prime}\right)$ for a $G^{\prime}$-stable $k^{\prime}$-submodule $W^{\prime}$ in $V^{\prime}$ (such a $W^{\prime}$ is clearly unique as a $k^{\prime}$-submodule, even without reference to the $G^{\prime}$-stability condition) and that $\bar{\Gamma}$ contains a $\left(V / V_{0}\right)(k)$-conjugate of $G$ (or equivalently that some $V(k)$-conjugate of $\Gamma$ in $G \ltimes V$ contains $G$ ).

Let $T \subseteq \Gamma$ be a maximal $k$-torus, so its image in $G$ is a maximal $k$-torus (as $\Gamma \rightarrow G$ is surjective). The maximal tori in $G \ltimes V$ are conjugates of maximal tori of $G$ since $V$ is unipotent, so by dimension reasons it follows that $T$ is maximal as a $k$-torus of $G \ltimes V$. It is harmless to replace $\Gamma$ with a $(G \ltimes V)(k)$-conjugate since we aim to show that some $V(k)$-conjugate of $\Gamma$ contains $G$, and by a result of Grothendieck all maximal $k$-tori in $G \ltimes V$ are $k$-rationally conjugate since $k=k_{s}$ (see [8, Prop. A.2.10] for a self-contained elementary proof). Thus, we may arrange that $T$ coincides with any desired maximal $k$-torus of $G=\mathrm{R}_{k^{\prime} / k}\left(G^{\prime}\right)$ inside of $G \ltimes V$. Fix such a $T$. Note that now $T$ contains $Z$, as all maximal $k$-tori in $G$ must contain any central $k$-torus (such as $Z$ ).

The quotient map $\Gamma \rightarrow G$ must carry the Cartan $k$-subgroup $Z_{\Gamma}(T)$ onto $Z_{G}(T)$. By [8, Prop. A.5.15], there is a unique maximal $k^{\prime}$-torus $T^{\prime}$ in $G^{\prime}$ such that $T \subseteq$ $\mathrm{R}_{k^{\prime} / k}\left(T^{\prime}\right)$, and moreover $Z_{G}(T)=Z_{G}\left(\mathrm{R}_{k^{\prime} / k}\left(T^{\prime}\right)\right)=\mathrm{R}_{k^{\prime} / k}\left(T^{\prime}\right)$ (the final equality by comparison of $k$-points, as $Z_{G}(T)$ is smooth). Thus, $\operatorname{dim} Z_{\Gamma}(T) \geq \operatorname{dim} \mathrm{R}_{k^{\prime} / k}\left(T^{\prime}\right)$. 
But

$$
Z_{\Gamma}(T) \subseteq Z_{G \ltimes V}(T)=Z_{G}(T)
$$

since $V^{T}=0$ (as $V^{Z}=0$ and $Z \subseteq T$ ). Hence, $Z_{\Gamma}(T)=\mathrm{R}_{k^{\prime} / k}\left(T^{\prime}\right)$ inside of $G \ltimes V$. In particular, $\mathrm{R}_{k^{\prime} / k}\left(T^{\prime}\right) \subseteq \Gamma$, so $\mathrm{R}_{k^{\prime} / k}\left(Z^{\prime}\right) \subseteq \Gamma$. Hence, the $k$-subgroup $V_{0}$ in $V$ is an $\mathrm{R}_{k^{\prime} / k}\left(Z^{\prime}\right)$-stable closed $k$-subgroup of $V$. The structure of $V_{0}$ is therefore determined by:

Lemma A.2.3. Let $k$ be a field, $k^{\prime}$ a nonzero finite reduced $k$-algebra, and $V^{\prime}$ a finitely generated $k^{\prime}$-module equipped with a linear action by a $k^{\prime}$-torus $Z^{\prime}$ such that the action of the Lie algebra $\mathfrak{z}^{\prime}=\operatorname{Lie}\left(Z^{\prime}\right)$ on $V^{\prime}$ satisfies $V^{\prime \mathfrak{z}^{\prime}}=0$. Any $R_{k^{\prime} / k}\left(Z^{\prime}\right)$ stable smooth closed $k$-subgroup $H$ of $V:=R_{k^{\prime} / k}\left(V^{\prime}\right)$ has the form $R_{k^{\prime} / k}\left(W^{\prime}\right)$ for a unique $k^{\prime}$-submodule $W^{\prime}$ of $V^{\prime}$.

As in Remark A.1.4 the hypothesis on the $\mathfrak{z}^{\prime}$-action cannot be dropped. The proof of Lemma A.2.3 is rather long, so let us first see how to use it to complete the proof of Proposition A.1.3.

We write $k^{\prime}=\prod k_{i}^{\prime}$ for fields $k_{i}^{\prime}$, and correspondingly $V^{\prime}=\prod V_{i}^{\prime}$ for a vector space $V_{i}^{\prime}$ over $k_{i}^{\prime}$. Let $G_{i}^{\prime}$ denote the $k_{i}^{\prime}$-fiber of $G^{\prime}$ (so $G=\prod \mathrm{R}_{k_{i}^{\prime} / k}\left(G_{i}^{\prime}\right)$ ). The $k^{\prime}$-torus $T^{\prime}$ contains the central torus $Z^{\prime}$, and we recall that $V^{\prime Z^{\prime}}=0$. Granting Lemma A.2.3, the Zariski closure $V_{0}$ of $(\Gamma \cap V)(k)$ in $V$ is $\mathrm{R}_{k^{\prime} / k}\left(W^{\prime}\right)$ for a unique $k^{\prime}$-submodule $W^{\prime}$ of $V^{\prime}$.

Note that $V_{0}$ is $G$-stable, due to the surjectivity of $\Gamma \rightarrow G$, so consideration of $k$-points shows that $W^{\prime}$ is a $G^{\prime}$-stable $k^{\prime}$-submodule of $V^{\prime}$ (as $k=k_{s}$ ). Since $V_{0}(k)=(\Gamma \cap V)(k)$, we see that $\Gamma(k) \cap V(k)$ coincides with the $k^{\prime}$-submodule $W^{\prime}$ of $V^{\prime}$. The $k$-group $\Gamma$ is therefore the full preimage in $G \ltimes V$ of a smooth closed $k$-subgroup $\bar{\Gamma}$ in $G \ltimes\left(V / V_{0}\right)$ such that $\bar{\Gamma}(k) \cap\left(V / V_{0}\right)(k)=0$. Let $\bar{V}=V / V_{0}$.

The maximal central torus $Z$ of $\mathrm{R}_{k^{\prime} / k}\left(Z^{\prime}\right)$ satisfies $V^{Z}=0$. Hence, $\bar{V}^{Z}=0$ as well, since $Z$ is a torus. For any $\gamma=(g, \bar{v}) \in \bar{\Gamma}(k)$ the effect of $\gamma^{-1}$-conjugation on an element $z \in Z(k)$ is the same as conjugation by $\bar{v}^{-1}=-\bar{v}$. Thus, $\gamma^{-1} z \gamma=$ $\bar{v}^{-1} z \bar{v}=(z, \bar{v}-z \bar{v})$, so since $Z(k) \subseteq \bar{\Gamma}(k)$ we have $z \bar{v}-\bar{v} \in \bar{\Gamma}(k) \cap \bar{V}(k)=0$. Letting $z$ vary, it follows that $\bar{v} \in \bar{V}^{Z}=0$. In other words, $\gamma \in G(k)$ inside of $(G \ltimes V)(k)$. That is, $\bar{\Gamma} \subseteq G$ inside of $G \ltimes V$. But the projection $\bar{\Gamma} \rightarrow G$ is surjective, so $\bar{\Gamma}=G$ inside of $G \ltimes \bar{V}$, as required. This completes the proof of Proposition A.1.3. conditional on Lemma A.2.3.

Proof of Lemma A.2.3. By Galois descent (in view of the uniqueness claim), we may and do assume $k=k_{s}$. The uniqueness of $W^{\prime}$ is clear, so the problem is its existence. Let $\left\{k_{i}^{\prime}\right\}$ be the set of factor fields of $k^{\prime}$, and $Z_{i}^{\prime}$ the $k_{i}^{\prime}$-fiber of $Z^{\prime}$. Consider the $k$-group decomposition $\mathrm{R}_{k^{\prime} / k}\left(Z^{\prime}\right)=\prod \mathrm{R}_{k_{i}^{\prime} / k}\left(Z_{i}^{\prime}\right)$, so the maximal $k$ torus $Z$ of $\mathrm{R}_{k^{\prime} / k}\left(Z^{\prime}\right)$ has the form $\prod Z_{i}$ for the maximal $k$-torus $Z_{i}$ in $\mathrm{R}_{k_{i}^{\prime} / k}\left(Z_{i}^{\prime}\right)$. Note that the character groups of $Z_{i}^{\prime}$ and $Z_{i}$ naturally coincide since $k_{i}^{\prime} / k$ is purely inseparable (and $k=k_{s}$ ). We may replace $H$ with $H^{0}$, so $H$ is connected. Our goal is to show that $H$ contains the image of its projection into each $\mathrm{R}_{k_{i}^{\prime} / k}\left(V_{i}^{\prime}\right)$.

Choose an index $i_{0}$ and let $S=\prod_{i \neq i_{0}} \mathrm{R}_{k_{i}^{\prime} / k}\left(Z_{i}^{\prime}\right)$, so $V^{S}=\mathrm{R}_{k_{i_{0}}^{\prime} / k}\left(V_{i_{0}}^{\prime}\right)$, where $V_{i}^{\prime}$ is the $k_{i}^{\prime}$-factor of $V^{\prime}$. The centralizer $H^{S}$ for the $S$-action on $H$ is smooth and connected (since $S$ is a torus and $H$ is smooth and connected), and the image of $H^{S}$ in $\mathrm{R}_{k_{i_{0}}^{\prime} / k}\left(V_{i_{0}}^{\prime}\right)$ is the same as the image of $H$ since the formation of $S$ invariants commutes with the formation of images under homomorphisms between 
smooth connected affine $k$-groups. Thus, we can replace $\left(k^{\prime} / k, V^{\prime}, Z^{\prime}, H\right)$ with $\left(k_{i_{0}}^{\prime} / k, V_{i_{0}}^{\prime}, Z_{i_{0}}^{\prime}, H^{S}\right)$ to reduce to the case that $k^{\prime}$ is a field.

With $k^{\prime}$ now arranged to be a field, we will show that $H$ contains the image of its projection into each $\mathrm{R}_{k^{\prime} / k}\left(V_{\chi^{\prime}}^{\prime}\right)$, with $V_{\chi^{\prime}}^{\prime}$ varying through the $Z^{\prime}$-weight spaces of $V^{\prime}$. Pick $\chi_{0}^{\prime}$ such that $V_{\chi_{0}^{\prime}}^{\prime} \neq 0$, and let $T^{\prime}=\left(\operatorname{ker} \chi_{0}^{\prime}\right)_{\text {red }}^{0}$ be the codimension-1 torus in $Z^{\prime}$ killed by $\chi_{0}^{\prime}$. Let $T$ be the maximal $k$-torus in $\mathrm{R}_{k^{\prime} / k}\left(T^{\prime}\right)$, so $\mathrm{X}(T)$ is naturally identified with $\mathrm{X}\left(T^{\prime}\right)$. Clearly $V^{T}=\mathrm{R}_{k^{\prime} / k}\left(V^{T^{\prime}}\right)$, and this is the direct product of the factors $\mathrm{R}_{k^{\prime} / k}\left(V_{\psi^{\prime}}^{\prime}\right)$, where $\psi^{\prime} \in \mathrm{X}\left(Z^{\prime} / T^{\prime}\right) \simeq \mathbf{Z}$ varies through those $\chi^{\prime}$ that are rational multiples of $\chi_{0}^{\prime}$. Also, the $T$-centralizer $H^{T}$ is smooth and connected (as for a torus action on any smooth connected affine group), and $H^{T} \rightarrow \mathrm{R}_{k^{\prime} / k}\left(V_{\chi_{0}^{\prime}}^{\prime}\right)$ has the same image as $H$ (since $T$ acts trivially on $\mathrm{R}_{k^{\prime} / k}\left(V_{\chi_{0}^{\prime}}^{\prime}\right)$ ). Since $\mathrm{X}\left(Z^{\prime} / T^{\prime}\right)$ is saturated in $\mathrm{X}\left(Z^{\prime}\right)$, we may replace $\left(V^{\prime}, Z^{\prime}, H\right)$ with $\left(V^{\prime T^{\prime}}, Z^{\prime} / T^{\prime}, H^{T}\right)$ to reduce to the case that $Z^{\prime} \simeq \mathrm{GL}_{1}$.

The image of $H$ in each $\mathrm{R}_{k^{\prime} / k}\left(V_{\chi^{\prime}}^{\prime}\right)$ has as its group of $k$-points an additive subgroup $W_{\chi^{\prime}}^{\prime}$ of $V_{\chi^{\prime}}^{\prime}$ stable under the action of $\mathrm{R}_{k^{\prime} / k}\left(Z^{\prime}\right)(k)=k^{\prime \times}$ through $\chi^{\prime}$. That is, writing $\chi^{\prime}(t)=t^{m}$ with $\operatorname{char}(k) \nmid m$ (due to our hypothesis on the vanishing of the $\mathfrak{z}^{\prime}$-invariants), the surjectivity of the $m$ th-power endomorphism of $k^{\prime \times}$ implies that $W_{\chi^{\prime}}^{\prime}$ is a $k^{\prime}$-linear subspace of $V_{\chi^{\prime}}^{\prime}$ for every $\chi^{\prime}$. We can replace $V_{\chi^{\prime}}^{\prime}$ with $W_{\chi^{\prime}}^{\prime}$ for every $\chi^{\prime}$ to arrange that $H \rightarrow \mathrm{R}_{k^{\prime} / k}\left(V_{\chi^{\prime}}^{\prime}\right)$ is surjective for all $\chi^{\prime}$. Now we aim to prove that $H=V$; i.e., $H$ contains $\mathrm{R}_{k^{\prime} / k}\left(V_{\chi^{\prime}}^{\prime}\right)$ for all $\chi^{\prime}$.

Fix a choice of $\chi_{0}^{\prime}$. The idea is to find a functorial way of selecting a $Z$-stable smooth closed $k$-subgroup $F(G)$ of any smooth affine $k$-group $G$ equipped with a $Z$-action so that the following formal properties hold: (i) $F(V)=\mathrm{R}_{k^{\prime} / k}\left(V_{\chi_{0}^{\prime}}^{\prime}\right)$ (avoiding any reference to the linear structure on $V^{\prime}$ over $k^{\prime}$ or the linear structure on $V$ over $k$ !); (ii) $F$ is a "projector" in the sense that $F(F(G))=F(G)$ for any $G$; (iii) $F$ carries surjections to surjections (without smoothness hypotheses on these surjections). Once such an $F$ is in hand, applying it to the $Z$-equivariant quotient map $H \rightarrow \mathrm{R}_{k^{\prime} / k}\left(V_{\chi_{0}^{\prime}}^{\prime}\right)=F(V)$ yields a surjective map $F(H) \rightarrow F(F(V))=F(V)$, yet $H$ is a $Z$-stable closed $k$-subgroup of $V$, so by functoriality of the subgroup assignment $F$ we see that $F(H)$ is a $k$-subgroup of $F(V)$ inside of $V$. In other words, $F(H)$ is a $k$-subgroup of $F(V)=\mathrm{R}_{k^{\prime} / k}\left(V^{\prime}\right)$ inside of $V$ such that the natural projection $V \rightarrow \mathrm{R}_{k^{\prime} / k}\left(V_{\chi_{0}^{\prime}}^{\prime}\right)=F(V)$ extending the identity on $F(V)$ restricts to a surjection on $F(H)$, forcing $F(H)=F(V)$. This implies that $H$ contains $F(H)=$ $F(V)=\mathrm{R}_{k^{\prime} / k}\left(V_{\chi_{0}^{\prime}}^{\prime}\right)$ inside of $V$, as desired.

We will not quite find such a functor $F$ in the generality just described. Instead, we will first carry out some preliminary reduction steps to acquire finer properties for $\chi_{0}^{\prime}$ (in comparison with all other $Z^{\prime}$-weights on $V^{\prime}$ ), and then we will construct such an $F$. Our aim is to prove that $H$ contains $\mathrm{R}_{k^{\prime} / k}\left(V_{\chi_{0}^{\prime}}^{\prime}\right)$ for an arbitrary but fixed choice of $\chi_{0}^{\prime}$, so we begin by composing the isomorphism $Z^{\prime} \simeq \mathrm{GL}_{1}$ with inversion if necessary to ensure that $\chi_{0}^{\prime}(t)=t^{n}$ with $n>0$. Proceeding by descending induction (or by contradiction), we may and do assume that $H$ contains $\mathrm{R}_{k^{\prime} / k}\left(V_{\chi^{\prime}}^{\prime}\right)$ for any $\chi^{\prime}: t \mapsto t^{m}$ with $m>n$. It is clearly harmless to pass to the quotient of $V^{\prime}$ by the span of such $V_{\chi^{\prime}}^{\prime}$ with "weight" larger than $n$ (and pass to the quotient of $H$ by the $k$-subgroup directly spanned by the $\mathrm{R}_{k^{\prime} / k}\left(V_{\chi^{\prime}}^{\prime}\right)$ for such $\left.\chi^{\prime}\right)$, so now every positive weight $\chi^{\prime}$ that occurs in $V^{\prime}$ has the form $\chi^{\prime}(t)=t^{m}$ with $m \leq n$. 
The maximal $k$-torus $Z$ in $\mathrm{R}_{k^{\prime} / k}\left(Z^{\prime}\right)=\mathrm{R}_{k^{\prime} / k}\left(\mathrm{GL}_{1}\right)$ is the evident $\mathrm{GL}_{1}$. Since the $k^{\prime}$-group $Z^{\prime}[n]=\mu_{n}$ is finite étale (as $\left.\operatorname{char}(k) \nmid n\right)$ and $k^{\prime} / k$ is purely inseparable, clearly $\mathrm{R}_{k^{\prime} / k}\left(Z^{\prime}[n]\right)=Z[n]$ and $V^{Z[n]}=\mathrm{R}_{k^{\prime} / k}\left(V^{Z^{\prime}[n]}\right)$. Thus, exactness of the formation of $\mu_{n}$-invariants allows us to replace $Z^{\prime}$ with $Z^{\prime} / Z^{\prime}[n]$, replace $V^{\prime}$ with $V^{\prime Z^{\prime}[n]}$ (which leaves $V_{\chi_{0}^{\prime}}^{\prime}$ unchanged), and replace $H$ with $\left(H^{\mu_{n}}\right)^{0}$. This brings us to the case that $\chi_{0}^{\prime}(t)=t$ and all other weights are negative. In other words, each $\chi^{\prime} \neq \chi_{0}^{\prime}$ satisfying $V_{\chi^{\prime}}^{\prime} \neq 0$ necessarily has the form $\chi^{\prime}=\chi_{0}^{\prime m}$ for some $m<0$ (depending on $\chi^{\prime}$ ).

Finally, we bring in a procedure that compatibly separates positive weights from negative weights in both $H$ and $\mathrm{R}_{k^{\prime} / k}\left(V^{\prime}\right)$ under the action of $Z=\mathrm{GL}_{1}$. The basic construction we need is systematically developed in [8, 2.1] in a functorial manner, and it goes as follows. By [8, Rem. 2.1.11], for any affine $k$-group scheme $G$ of finite type and left action $\mu$ of $\mathrm{GL}_{1}$ on $G$, there is a closed $k$-subgroup scheme $U_{G}(\mu)$ of $G$ representing the functor that assigns to any $k$-algebra $R$ the subgroup of points $g \in G(R)$ such that the $R$-scheme morphism $\mathrm{GL}_{1} \rightarrow G_{R}$ defined by $t \mapsto t . g$ extends (necessarily uniquely) to an $R$-scheme morphism $\mathbf{A}_{R}^{1} \rightarrow G_{R}$. More specifically, by [8. Rem. 2.1.11], $U_{G}(\mu)$ is smooth and connected when $G$ is smooth, and if $G \rightarrow \bar{G}$ is a $\mathrm{GL}_{1}$-equivariant flat surjection between connected affine $k$-groups of finite type then $U_{G}(\mu) \rightarrow U_{\bar{G}}(\mu)$ is a flat quotient map. Note also that if $G^{\prime}$ is a closed $k$ subgroup of $G$ that is stable under the action of $\mathrm{GL}_{1}$ then $U_{G^{\prime}}(\mu)=G^{\prime} \cap U_{G}(\mu)$. The functor $(G, \mu) \rightsquigarrow U_{G}(\mu)$ will play the role of the functor $F$ in the formal considerations given above.

Define the isomorphism $\lambda: \mathrm{GL}_{1} \simeq Z$ to be the inverse of the preferred isomorphism used above (i.e., the inverse of the restriction of $\mathrm{R}_{k^{\prime} / k}\left(\chi_{0}^{\prime}\right): \mathrm{R}_{k^{\prime} / k}\left(Z^{\prime}\right) \simeq$ $\mathrm{R}_{k^{\prime} / k}\left(\mathrm{GL}_{1}\right)$ to maximal $k$-tori), and use this to identify the natural $Z$-action on $V$ with a $\mathrm{GL}_{1}$-action. (This is exactly the natural scaling action arising from the evident linear structure on $V=\mathrm{R}_{k^{\prime} / k}\left(V^{\prime}\right)$ over $k$, in view of how we have changed $Z^{\prime}$ in relation to $\chi_{0}^{\prime}$.) In this way we get smooth connected $k$-subgroups $U_{H}(\lambda)$ in $H$ and $U_{V}(\lambda)$ in $V$, with $U_{H}(\lambda)=H \cap U_{V}(\lambda)$ scheme-theoretically. Every $\chi^{\prime} \neq \chi_{0}^{\prime}$ such that $V_{\chi^{\prime}}^{\prime} \neq 0$ necessarily has the form $\chi_{0}^{\prime m}$ with $m<0$ (depending on $\chi^{\prime}$ ), so $U_{V}(\lambda)=\mathrm{R}_{k^{\prime} / k}\left(V_{\chi_{0}^{\prime}}^{\prime}\right)$. In particular, the $k$-subgroup $\bar{H}:=\mathrm{R}_{k^{\prime} / k}\left(V_{\chi_{0}^{\prime}}^{\prime}\right)$ in $V$ is stable under the $\mathrm{GL}_{1}$-action through $\lambda$ and satisfies $U_{\bar{H}}(\lambda)=\bar{H}$.

Since we reduced to the case that the composite map $H \hookrightarrow V \rightarrow \mathrm{R}_{k^{\prime} / k}\left(V_{\chi^{\prime}}^{\prime}\right)$ is surjective for all $\chi^{\prime}$, by taking $\chi^{\prime}=\chi_{0}^{\prime}$ we deduce that the $\mathrm{R}_{k^{\prime} / k}\left(Z^{\prime}\right)$-equivariant map $H \rightarrow \bar{H}$ is a $\mathrm{GL}_{1}$-equivariant flat quotient map. Thus, the induced map $U_{H}(\lambda) \rightarrow U_{\bar{H}}(\lambda)=\bar{H}$ is surjective. In other words, the surjective composite map $H \hookrightarrow \mathrm{R}_{k^{\prime} / k}\left(V^{\prime}\right) \rightarrow \mathrm{R}_{k^{\prime} / k}\left(V_{\chi_{0}^{\prime}}^{\prime}\right)$ restricts to a surjective map on the $k$-subgroup $U_{H}(\lambda)$. But $U_{H}(\lambda)$ viewed inside of $V$ is a $k$-subgroup of $U_{V}(\lambda)=\mathrm{R}_{k^{\prime} / k}\left(V_{\chi_{0}^{\prime}}^{\prime}\right)$, so we conclude that the inclusion $U_{H}(\lambda) \hookrightarrow \mathrm{R}_{k^{\prime} / k}\left(V_{\chi_{0}^{\prime}}^{\prime}\right)$ is an equality (since the natural projection $V \rightarrow \mathrm{R}_{k^{\prime} / k}\left(V_{\chi_{0}^{\prime}}^{\prime}\right)$ restricts to the identity on the $k$-subgroup $\mathrm{R}_{k^{\prime} / k}\left(V_{\chi_{0}^{\prime}}^{\prime}\right)$ ). This shows that the $k$-subgroup $H$ in $V$ contains $\mathrm{R}_{k^{\prime} / k}\left(V_{\chi_{0}^{\prime}}^{\prime}\right)$, as required.

A.3. A generalization. In the general setting introduced before Example A.1.2 there is no "linear structure" imposed on $V$ or the $G$-action on $V$. In that generality we have the following replacement for Proposition A.1.3 away from characteristic 2: 
Theorem A.3.1. Using the notation and hypotheses as at the start of $\$$ A.1, assume $\operatorname{char}(k) \neq 2$ and $V^{T}=0$ for some maximal $k$-torus $T$ of $G$. Let $\Gamma$ be a smooth closed $k$-subgroup of $G \ltimes V$ such that the composite map $\Gamma \rightarrow G$ is surjective.

The scheme-theoretic intersection $V_{0}:=\Gamma \cap V$ is smooth and $G$-stable, and there is a unique $v \in\left(V / V_{0}\right)(k)$ such that $\Gamma$ is the preimage under $G \ltimes V \rightarrow G \ltimes\left(V / V_{0}\right)$ of the v-conjugate of $G$ in $G \ltimes\left(V / V_{0}\right)$.

The proof of Theorem A.3.1 will appear elsewhere, as it involves techniques of an entirely different nature from the proof of Proposition A.1.3 the structure theory of pseudo-reductive groups developed in [8] (even though the statement of Theorem A.3.1 does not involve pseudo-reductive groups).

Theorem A.3.1 is probably valid in characteristic 2 under additional restrictions, including that the maximal geometric semisimple quotient $G_{\bar{k}}^{\mathrm{ss}}$ has no simple factor of type C. The necessity of additional hypotheses related to type $\mathrm{C}$ in a characteristic-2 version of Theorem A.3.1 is not merely a matter of technique, but rather is due to explicit counterexamples. Such counterexamples illuminate the meaning of Theorem A.3.1 and help one to appreciate the good fortune of the characteristic-free nature of Proposition A.1.3 so we now provide a family of counterexamples to a characteristic- 2 version of Theorem A.3.1 without type-C restrictions.

Example A.3.2. Let $k$ be a field of characteristic 2, and $(V, q)$ a non-degenerate quadratic space of rank $2 n+1$ over $k$. The associated symmetric bilinear form $B_{q}$ on $V$ is alternating with $V^{\perp}$ of dimension 1 , so there is an induced non-degenerate alternating form $\bar{B}_{q}$ on the quotient $\bar{V}=V / V^{\perp}$ of dimension $2 n$. Thus, we get a $\operatorname{map} \mathrm{SO}(q) \rightarrow \mathrm{Sp}\left(\bar{B}_{q}\right)$.

For example, if $q=x_{0}^{2}+x_{1} x_{2}+\cdots+x_{2 n-1} x_{2 n}$ is the standard split quadratic form on $V=k^{2 n+1}$ then $V^{\perp}=k e_{0}$ and the map

$$
\mathrm{SO}_{2 n+1}=\mathrm{SO}(q) \rightarrow \mathrm{Sp}\left(\bar{B}_{q}\right)=\mathrm{Sp}_{2 n}
$$

is projection onto the lower-right $2 n \times 2 n$ block matrix. This map is surjective and its kernel consists of lower-triangular unipotent matrices whose entries below the diagonal all vanish apart from $\alpha_{2}$ entries along the left column (below the upper-left entry of 1). As a group scheme, this kernel is $\alpha_{2}^{2 n}$ with components corresponding to matrix entries along the left column, and the induced action on it by $\mathrm{Sp}_{2 n}$ is via the identification of $\alpha_{2}^{2 n}$ as the Frobenius kernel in the standard representation space of rank $2 n$. (To put this in perspective, we note that by [21, Lemma 2.2], over an algebraically closed field the only isogenies between absolutely simple and connected semisimple groups for which the kernel is nontrivial and unipotent are the isogenies (A.3.3) in characteristic 2, up to an isomorphism on the source and target.)

In general, let $W$ denote the vector space $\operatorname{Hom}\left(V^{\perp}, \bar{V}\right)$ corresponding to "left column below the top entry" in $\mathfrak{s o}_{2 n+1} \subset \mathfrak{g l}_{2 n+1}$, and equip it with the standard action by $\operatorname{Sp}\left(\bar{B}_{q}\right) \subset \mathrm{GL}(\bar{V})$. Viewing $W$ as a vector group over $k$, let $F_{W / k}: W \rightarrow$ $W^{(2)}$ denote its relative Frobenius isogeny. There is an exact sequence

$$
1 \rightarrow \operatorname{ker} F_{W / k} \rightarrow \mathrm{SO}(q) \rightarrow \mathrm{Sp}\left(\bar{B}_{q}\right) \rightarrow 1
$$


in which the induced left action of the quotient term on the commutative kernel is the natural one arising from the $\operatorname{Sp}\left(\bar{B}_{q}\right)$-action on $W$. The pushout along the $\operatorname{Sp}\left(\bar{B}_{q}\right)$-equivariant inclusion $\operatorname{ker} F_{W / k} \hookrightarrow W$ is an exact sequence

$$
1 \rightarrow W \rightarrow E \rightarrow \operatorname{Sp}\left(\bar{B}_{q}\right) \rightarrow 1
$$

in which $E$ contains the subgroup $\Gamma:=\mathrm{SO}(q)$ mapping via a degree- $2^{2 n}$ infinitesimal isogeny onto $\operatorname{Sp}\left(\bar{B}_{q}\right)$ and the induced left action of $\operatorname{Sp}\left(\bar{B}_{q}\right) \subset \operatorname{GL}(\bar{V})$ on the commutative kernel $W=\operatorname{Hom}\left(V^{\perp}, \bar{V}\right)$ is the natural one. (By choosing a basis of the line $V^{\perp}$, we get an $\operatorname{Sp}\left(\bar{B}_{q}\right)$-equivariant isomorphism $W \simeq \bar{V}$.) Thus, for any maximal torus $T$ in $\operatorname{Sp}\left(\bar{B}_{q}\right)$ the subspace $W^{T}$ vanishes because the center $\mu_{2}$ of the symplectic group acts by ordinary scaling (and so even $W^{\mu_{2}}$ vanishes). We claim that (A.3.4) splits as a semidirect product, so inside of $\operatorname{Sp}\left(\bar{B}_{q}\right) \ltimes \bar{V}$ we get a counterexample to the conclusion of Theorem A.3.1 in type-C cases in characteristic 2 .

To construct a splitting of (A.3.4), more generally consider an arbitrary short exact sequence

$$
1 \rightarrow U \rightarrow E \rightarrow \mathrm{Sp}_{2 n} \rightarrow 1
$$

of linear algebraic groups over a field $k$, where $U \simeq \mathbf{G}_{\mathrm{a}}^{N}$ is a vector group. Assume that the induced action of the center $\mu=\mu_{2}$ of $\mathrm{Sp}_{2 n}$ on $U$ is the natural scaling action. We shall prove that (A.3.5) is split.

Note that $E$ is necessarily a smooth connected affine $k$-group. The exact sequence A.3.5 pulls back to an extension of $\mu$ by $U$ lifting the natural linear action of $\mu$ on $\mathbf{G}_{\mathrm{a}}^{N}$. We claim that this pullback splits, so $\mu$ lifts into $E$. To prove that the pointed set $\operatorname{Ex}_{k}\left(\mu_{2}, \mathbf{G}_{\mathrm{a}}^{N}\right)$ of such extensions is trivial, we immediately reduce to the case $N=1$. The case $\operatorname{char}(k) \neq 2$ is easy (since then $\mu_{2}=\mathbf{Z} / 2 \mathbf{Z}$ and doubling is an automorphism of $\mathbf{G}_{\mathrm{a}}$ ). Suppose instead that $\operatorname{char}(k)=2$. Then consideration of Frobenius kernels reduces the problem to the vanishing of $\operatorname{Ex}_{k}\left(\mu_{2}, \alpha_{2}\right)$ over fields of characteristic $p=2$ (relative to the usual scaling action of $\mu_{2}$ on $\alpha_{2}$ ), and this in turn follows from an easy calculation with $p$-Lie algebras (and works just as well with $\mu_{p}$ and $\alpha_{p}$ for $p>2$ ).

For a $k$-subgroup $\mu^{\prime} \subset E$ isomorphically lifting the center $\mu$ of $\mathrm{Sp}_{2 n}$, consider the scheme-theoretic centralizer $E^{\mu^{\prime}}$ of $\mu^{\prime}$ in $E$. By [8, Prop. A.8.10], $E^{\mu^{\prime}}$ is smooth and $\operatorname{Lie}\left(E^{\mu^{\prime}}\right)$ is equal to $\operatorname{Lie}(E)^{\mu^{\prime}}$. By exactness of $\mu^{\prime}$-invariants on linear representations, the surjective map $\operatorname{Lie}(E) \rightarrow \mathfrak{s p}_{2 n}$ (with kernel $\operatorname{Lie}(U)$ ) induces a surjective map Lie $(E)^{\mu^{\prime}} \rightarrow \mathfrak{s p}_{2 n}$. This latter surjection is an isomorphism since its kernel is $\operatorname{Lie}(U)^{\mu^{\prime}}=\operatorname{Lie}\left(\mathbf{G}_{\mathrm{a}}^{N}\right)^{\mu}=\left(\operatorname{Lie}\left(\mathbf{G}_{\mathrm{a}}\right)^{\mu}\right)^{N}=0$. Hence, $\left(E^{\mu^{\prime}}\right)^{0} \rightarrow \mathrm{Sp}_{2 n}$ is an isogeny with étale kernel. But the connected semisimple group $\mathrm{Sp}_{2 n}$ is simply connected, so $\left(E^{\mu^{\prime}}\right)^{0} \rightarrow \mathrm{Sp}_{2 n}$ is an isomorphism; this is the required splitting.

\section{ACKNOWLEDGEMENTS}

The authors thank J. Yu for many helpful discussions and suggestions and for encouragement throughout this project. They particularly thank NCTS for financial support so that they were able to visit each other over the past several years. The authors further thank B. Conrad for providing them with the appendix, crucially helping them handle inseparability issues occurring in the case of quasi-logarithms. Finally, they thank the referees for several helpful suggestions. 


\section{REFERENCES}

1. G. W. Anderson, t-motives, Duke Math. J. 53 (1986), 457-502. MR850546 (87j:11042)

2. G. W. Anderson, W. D. Brownawell, and M. A. Papanikolas, Determination of the algebraic relations among special $\Gamma$-values in positive characteristic, Ann. of Math. (2) 160 (2004), 237-313. MR2119721 (2005m:11140)

3. A. Baker and G. Wüstholz, Logarithmic forms and Diophantine geometry, Cambridge University Press, Cambridge, 2007. MR2382891(2009e:11001)

4. W. D. Brownawell, Minimal extensions of algebraic groups and linear independence, J. Number Theory 90 (2001), 239-254. MR1858075 (2002h:11066)

5. W. D. Brownawell and M. A. Papanikolas, Linear independence of Gamma-values in positive characteristic, J. reine angew. Math. 549 (2002), 91-148. MR:1916653 (2003g:11080)

6. C.-Y. Chang, On periods of the third kind for rank 2 Drinfeld modules, preprint.

7. C.-Y. Chang and M. A. Papanikolas, Algebraic relations among periods and logarithms of rank 2 Drinfeld modules, Amer. J. Math. 133 (2011), 359-391.

8. B. Conrad, O. Gabber, and G. Prasad, Pseudo-reductive groups, New Math. Monographs 17, Cambridge University Press, 2010. MR2723571

9. S. David and L. Denis, Périodes de modules de Drinfeld "l'indépendance quadratique en rang II," J. Ramanujan Math. Soc. 17 (2002), 65-83. MR1906421 (2003i:11103)

10. P. Deligne, J. S. Milne, A. Ogus, and K.-Y. Shih, Hodge cycles, motives, and Shimura varieties, Lecture Notes in Mathematics, vol. 900, Springer-Verlag, Berlin, 1982. MR654325 (84m:14046)

11. V. G. Drinfeld, Elliptic modules, Math. USSR-Sb. 23 (1974), 561-592. MR0384707 (52:5580)

12. B. Farb and R. K. Dennis, Noncommutative algebra, Springer-Verlag, New York, 1993. MR.1233388 (94j:16001)

13. E.-U. Gekeler, On the de Rham isomorphism for Drinfeld modules, J. reine angew. Math. 401 (1989), 188-208. MR.1018059 (90g:11070)

14. D. Goss, Basic structures of function field arithmetic, Springer-Verlag, Berlin, 1996. MR:1423131 (97i:11062)

15. C. Hardouin, Computation of the Galois groups occurring in M. Papanikolas's study of Carlitz logarithms, arXiv:0906.4429.

16. A.-K. Juschka, The Hodge conjecture for function fields, Diplomarbeit, Universität Münster, 2010.

17. M. A. Papanikolas, Tannakian duality for Anderson-Drinfeld motives and algebraic independence of Carlitz logarithms, Invent. Math. 171 (2008), 123-174. MR2358057 (2009b:11127)

18. F. Pellarin, Aspects de l'indépendance algébrique en caractéristique non nulle, Séminaire Bourbaki, Vol. 2006/2007, Astérisque No. 317 (2008), Exp. no. 973, viii, 205-242. MR2487735 (2010c:11086)

19. R. Pink, Hodge structures over function fields, preprint, 1997, http://www.math. ethz.ch/ pink/.

20. R. Pink, The Mumford-Tate conjecture for Drinfeld modules, Publ. Res. Inst. Math. Sci. 33 (1997), 393-425. MR.1474696 (98f:11062)

21. G. Prasad and J.-K. Yu, On quasi-reductive group schemes. With an appendix by B. Conrad, J. Algebraic Geom. 15 (2006), 507-549. MR2219847 (2007c:14047)

22. D. S. Thakur, Function field arithmetic, World Scientific Publishing, River Edge, NJ, 2004. MR2091265 (2005h:11115)

23. A. Thiery, Indépendance algébrique des périodes et quasi-périodes d'un module de Drinfeld, The arithmetic of function fields (Columbus, OH, 1991), de Gruyter, Berlin (1992), 265-284. MR.1196524 (93j:11034)

24. M. Waldschmidt, Elliptic functions and transcendence, Surveys in Number Theory, Dev. Math. 17 (2008), 143-188. MR 2462949 (2010a:11142)

25. J. Yu, Transcendence and Drinfeld modules, Invent. Math. 83 (1986), 507-517. MR827364 (87g:11088)

26. J. Yu, On periods and quasi-periods of Drinfeld modules, Compositio Math. 74 (1990), 235245. MR1055694(91i:11089)

27. J. Yu, Analytic homomorphisms into Drinfeld modules, Ann. of Math. (2) 145 (1997), 215233. MR1441876 (98c:11054) 
Department of Mathematics, National Tsing Hua University, No. 101, Sec. 2, Kuang Fu Road, Hsinchu City 30042, Taiwan, Republic of China and National Center for Theoretical Sciences, Hsinchu City 30042, Taiwan, Republic of China

E-mail address: cychang@math.cts.nthu.edu.tw

Department of Mathematics, Texas A\&M University, College Station, Texas 77843 U.S.A.

E-mail address: map@math.tamu.edu

Department of Mathematics, Stanford University, Stanford, California 94305 U.S.A.

E-mail address: conrad@math.stanford.edu 